\title{
Next-to-leading power threshold effects for inclusive and exclusive processes with final state jets
}

\author{
Melissa van Beekveld, ${ }^{a, b}$ Wim Beenakker, ${ }^{a, c}$ Eric Laenen $^{b, c, d}$ and Chris D. White \\ ${ }^{a}$ Theoretical High Energy Physics, Radboud University, \\ Nijmegen, Heyendaalseweg 135, 6525 AJ Nijmegen, The Netherlands \\ ${ }^{b}$ Nikhef, \\ Science Park 105, 1098 XG Amsterdam, The Netherlands \\ ${ }^{c}$ Institute of Physics, University of Amsterdam, \\ Science Park 904, 1018 XE Amsterdam, The Netherlands \\ ${ }^{d}$ ITF, Utrecht University, \\ Leuvenlaan 4, 3584 CE Utrecht, The Netherlands \\ ${ }^{e}$ Centre for Research in String Theory, School of Physics and Astronomy, \\ Queen Mary University of London, \\ 327 Mile End Road, London E1 4NS, U.K. \\ E-mail: mcbeekveld@gmail.com, w.beenakker@science.ru.nl, \\ eric.laenen@nikhef.nl, christopher.white@qmul.ac.uk
}

ABSTRACT: It is well known that cross-sections in perturbative QCD receive large corrections from soft and collinear radiation, which can be resummed to all orders in the coupling. Whether or not the universal properties of this radiation can be extended to next-to-leading power (NLP) in the threshold expansion has been the subject of much recent study. In particular, universal forms for next-to-leading order (NLO) cross-sections have been obtained for general colour-singlet production processes by considering only the emission of gluons. In this paper, we extend such formulae to processes containing final state jets, and show that the dominant NLP terms at NLO can be obtained using a similar prescription to the colour-singlet case. We furthermore consider the emission of soft quarks, which also leads to a class of universal NLP contributions at NLO. We illustrate our results using three different processes at NLO: deep-inelastic scattering, hadroproduction via electron-positron annihilation and prompt photon production.

KEYwords: Resummation, Perturbative QCD

ArXiv ePrint: 1905.08741 


\section{Contents}

1 Introduction 1

2 Universal NLO amplitudes for (next-to-)soft radiation 3

2.1 Radiation of (next-to-)soft gluons 4

2.2 Radiation of soft quarks 8

3 NLP contributions in DIS at NLO 11

4 NLP contributions in $e^{+} e^{-} \rightarrow$ jets at NLO 15

$5 \quad$ NLP cross-section for NLO prompt photon production $\quad 17$

$\begin{array}{lll}5.1 q \bar{q} \rightarrow g g \gamma \text { channel } & 18\end{array}$

$5.2 q \bar{q} \rightarrow q \bar{q} \gamma$ channel $\quad 24$

$\begin{array}{lll}5.3 q g \rightarrow q g \gamma & 26\end{array}$

$\begin{array}{llr}6 & \text { Discussion } & 29\end{array}$

$\begin{array}{ll}\text { A Useful definitions } & 31\end{array}$

B Results for the remaining channels $\quad 32$

$\begin{array}{ll}\text { C NLO cross-section for prompt photon production } & 34\end{array}$

\section{Introduction}

The calculation of ever more precise results for cross-sections in perturbative Quantum Chromodynamics (QCD) is a perennial necessity for current and forthcoming collider experiments. Results typically proceed on two frontiers. Firstly, one must proceed to higher fixed orders in the strong coupling $\alpha_{s}$. Secondly, one must supplement fixed order calculations with contributions which are enhanced in certain kinematic regions. One such region is that of production of particles near threshold. In that case, the phase space for the emission of additional radiation is squeezed, leading to an incomplete cancellation between real and virtual singularities, and thus the appearance of large contributions at all orders in perturbation theory. More specifically, if $\xi$ is a dimensionless kinematic ratio such that $\xi \rightarrow 0$ near threshold, the corresponding differential cross-section has the following form

$$
\frac{\mathrm{d} \sigma}{\mathrm{d} \xi} \propto \sum_{n=0}^{\infty}\left(\frac{\alpha_{s}}{\pi}\right)^{n}\left[\sum_{m=0}^{2 n-1} c_{n m}^{R}\left(\frac{\ln ^{m} \xi}{\xi}\right)_{+}+c_{n}^{V} \delta(\xi)+\sum_{m=0}^{2 n-1} c_{n m}^{\mathrm{NLP}} \ln ^{m} \xi+\ldots\right] .
$$

The first two sets of terms originate from soft and collinear radiation (real or virtual). They make up the leading power (LP) contributions in the threshold variable $\xi$ and are localized at $\xi=0$. The contributions have a universal form, which allows for their all-order resummation [1-16]. 
The third set of terms in eq. (1.1) make up the next-to-leading power (NLP) contributions to the differential cross-section, as they are suppressed by a single power of the threshold variable. Although subleading, the increasing precision of both LP resummation and of experimental data makes such terms numerically relevant [17, 18]. As at LP, the highest power of the NLP logarithm at a given order is referred to as leading-logarithmic (LL). One may then worry about next-to-leading logarithmic (NLL) contributions, and so on. It may well be the case for collider processes of interest that the LL NLP terms (or beyond) must be resummed for an adequate comparison of theory with data. However, such terms can be equally important for estimating fixed-order calculations. Precision calculations are necessary for improving the modelling of SM backgrounds at the LHC, and there remain many such processes where higher-order results (analytic or numerical) are unavailable. Estimations of higher-order cross-sections based on the classification of NLP terms at fixed order in $\alpha_{s}$ may thus play a key role, analogous to how LP estimates have historically preceded exact next-to-leading-order (NLO) calculations. Furthermore, numerical codes (at NLO or beyond) require knowledge of soft radiation in infrared subtraction schemes. These schemes typically need the matching of these contributions in different phase-space configurations. Improving this matching to NLP promises to significantly extend the stability and speed of such codes [19-24].

The threshold expansion of eq. (1.1) contains contributions associated with radiation that is (next-to-)soft and/or collinear with an external parton leg. We will define the properties of such radiation more carefully in what follows, but here we note that much previous work has focused on the soft expansion, with the aim to classify its effects beyond LP. Next-to-soft radiation in gauge theory was first studied in the classic works of refs. [25, 26], and more recently in ref. [27]. Since then, a variety of approaches have been used to try to systematically elucidate the structure of next-to-soft corrections [28-38]. There has recently been a revival of interest in this topic, partially motivated by more formal work on so-called next-to-soft theorems of refs. [39, 40] (see also ref. [41]), which relates soft physics to asymptotic symmetries in gauge theory and gravity. This has led to a great deal of activity aiming to systematically classify NLP contributions to cross-sections, using either diagrammatic factorisation formulas that generalise their LP counterparts [42-46], or the framework of soft-collinear effective theory (SCET) [19, 47-56]. A resummation of LL NLP effects in Drell-Yan production is recently presented using the SCET approach [57] (see ref. [58] for a related study for other observables), confirming earlier expectations from refs. [30-34], and results using the diagrammatic approach are presented in ref. [59]. Here we restrict our attention to NLO in perturbation theory, similarly to how previous studies have focused on fixed-order effects [20, 22, 60-62]. Of particular relevance for the present study is ref. [60], which derived a universal form of the cross-section for the production of an arbitrary number of colour singlet particles at NLO, up to NLP level, in either the $q \bar{q}$ or gg channel. An especially elegant result was that the NLP cross-section can be expressed in terms of a simple kinematic shift of the LO result, where the specific form of this shift is dictated precisely by the next-to-soft theorems. This both illustrates the phenomenological use of next-to-soft factorisation formulas, and provides analytic information where this was previously absent (such as in di-Higgs production). All NLP terms are captured 
by the kinematic shift at this order in perturbation theory for these processes. This is due to the fact that no final state partons are present in the leading-order (LO) process, so that the threshold and soft expansions coincide. Indeed, most NLP studies effects (with the exception of the conjectural but well-motivated resummation proposal of refs. [30-34]) have focused on processes in which all real radiation is manifestly (next-to) soft, such as Drell-Yan production. It is then natural to ponder what the recently developed next-to-soft formalisms are able to capture, if instead final state jets are allowed to be present.

As discussed above, the presence of final state jets means that the threshold and nextto-soft expansions no longer coincide, in that the former contains collinear effects. However, it may well be that generalisations of the formulae derived in ref. [60] can be used to capture the LL NLP effects at NLO. We will see that this is indeed the case in what follows. Ref. [60] included only the effects of radiated gluons dressing the LO amplitude. However, at NLP one has the possibility to emit (anti-)quarks (e.g. see ref. [63]), which can also give rise to LL effects. We will show how the dominant terms can indeed be represented in a straightforward and universal way. To illustrate our results, we will use three examples of processes with final state jets: deep-inelastic scattering, quark-antiquark pair production in electron-positron annihilation, and the production of a photon in association with a hard coloured particle (prompt photon production).

This paper is structured as follows. In section 2 we derive an explicit expression for the NLO amplitude of a coloured final state in quark-antiquark, gluon-gluon or quark-gluon scattering, which includes dominant (leading-logarithmic) terms in the threshold expansion up to NLP level. This NLP amplitude can be subdivided into two separate contributions: a gluonic contribution (section 2.1) and a quark contribution (section 2.2). As in ref. [60], the amplitudes we obtain are fully general at NLO, and the results thus provide universal corrections to any Born process with massless coloured particles in the final state. We then illustrate how to apply our formalism in a number of examples of increasing complexity. In section 3 we consider deep-inelastic scattering, whose Born amplitude contains a single final-state parton, and in section 4 we examine hadroproduction in electron-positron annihilation, where two final state partons are present at LO. In section 5 we look at prompt photon production, which adds the complication of a final state which is not fully inclusive. In all cases, we find that leading logarithmic effects up to NLP are completely captured by performing a similar kinematic shift to that observed for colour singlet production processes [60], and in addition by the effects of soft quark radiation. Finally, in section 6 , we discuss the implications of our results before concluding.

\section{Universal NLO amplitudes for (next-to-)soft radiation}

As is well-known, radiation from a massless external line of an amplitude leads to infrared (IR) divergences, which in turn gives rise to threshold logarithms in the final cross-section. Given a radiated particle with 4-momentum $k$, a divergence will occur if this momentum is soft (all components going parametrically to zero), or collinear to the emitting particle momentum $p^{\mu}$. The particle may also be soft and collinear, in which case the divergence is maximal. Next-to-soft corrections then correspond to a systematic expansion of the entire 
amplitude in the momentum of the emitted radiation, keeping the first subleading order only. We will use the term hard-collinear to refer to a radiated particle that is collinear to an external parton in an amplitude, but not soft or next-to-soft. Without double-counting, we may then refer to radiated particles as either soft, next-to-soft or hard-collinear. ${ }^{1}$

For the production of massive colour singlet particles considered in ref. [60], the only IR divergences that appear at NLO are manifestly associated with (next-to-)soft radiation. More specifically, that study examined dressing the amplitude for production of $N$ massive colour singlet particles with an additional gluon, and derived a universal form for the NLO cross-section for any such process, valid up to next-to-soft order in the momentum of the emitted radiation. Here we will revisit this analysis when final state massless partons are present. As is well-known, the soft expansion then does not coincide with the threshold expansion of eq. (1.1), but must be supplemented by hard-collinear effects. However, as we will demonstrate explicitly, the leading-logarithmic terms at NLP stem from radiation that is next-to-soft. Thus, a similar approach to ref. [60] can be used to capture this class of NLO corrections.

For the emission of gluons, we will recover the results of refs. [25-27, 40]. In addition, the re-derivation presented here will allow us to set up a careful notation that is needed for what follows. A significant extension of previous results, however, is a universal nextto-soft amplitude for the NLO emission of soft quarks, which we present here for the first time. The latter effect is known to be absent at LP in the threshold expansion, but must be included at NLP level and beyond.

\subsection{Radiation of (next-to-)soft gluons}

Let us first consider the emission of (next-to-)soft gluons. We will do this for a generic Born level process with 2 initial state coloured particles and $n$ final state coloured particles (see figure 1a). As mentioned above, this extends the work of ref. [60] for colour-singlet production, itself based on the earlier work of refs. [45, 46] (see also refs. [42-44, 48, 65]). All particles are considered massless, which will be the case for all processes considered throughout the paper. ${ }^{2}$ We must then consider all possible ways in which a gluon can be emitted, namely the contributions of figure $1 \mathrm{~b}-1 \mathrm{f}$, where momenta and colour indices are defined as shown. The first of these contributions, figure 1b, yields the matrix element

$$
\begin{aligned}
i \mathcal{M}_{1, q}= & -\frac{i g_{s} t_{c_{j} c_{i}}^{c}}{\left(p_{1}-k\right)^{2}+i \varepsilon} \mathcal{M}_{c_{j}}\left(p_{1}-k, p_{2}, \ldots, p_{n+2}\right)\left(\not p_{1}-\not k\right) \gamma^{\sigma} u\left(p_{1}\right) \epsilon_{\sigma}^{*}(k) \\
= & -\frac{i g_{s} t_{c_{j} c_{i}}^{c}}{\left(p_{1}-k\right)^{2}+i \varepsilon} \mathcal{M}_{c_{j}}\left(p_{1}, p_{2}, \ldots, p_{n+2}\right) \\
& \times\left(\left(2 p_{1}^{\sigma}-k^{\sigma}\right)-2 i S^{\sigma \mu} k_{\mu}-2 k^{\mu} \frac{\overleftarrow{\partial}}{\partial p_{1}^{\mu}} p_{1}^{\sigma}\right) u\left(p_{1}\right) \epsilon_{\sigma}^{*}(k),
\end{aligned}
$$

where $\left\{t_{i j}^{a}\right\}$ are elements of the colour generator in the fundamental representation, and $\mathcal{M}_{c_{j}}$ is the hard scattering matrix element carrying colour label $c_{j}$ for the incoming quark.

\footnotetext{
${ }^{1} \mathrm{~A}$ systematic way to characterise radiated momenta is via the method of regions [64] for classifying threshold corrections, which has been applied recently to analyse NLP effects in refs. [61, 62].

${ }^{2}$ We note in passing that our formalism would also apply for massive coloured particles.
} 


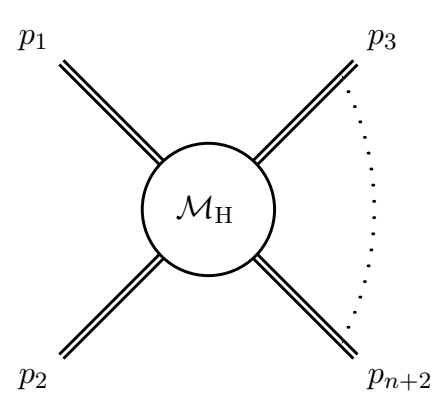

(a)

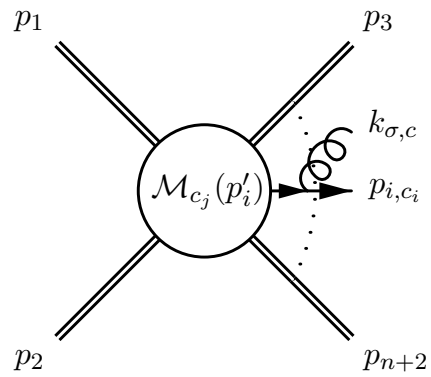

(d)

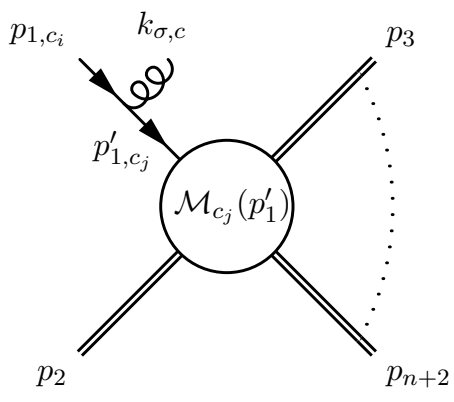

(b)

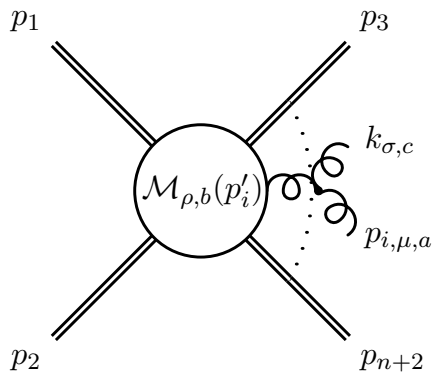

(e)

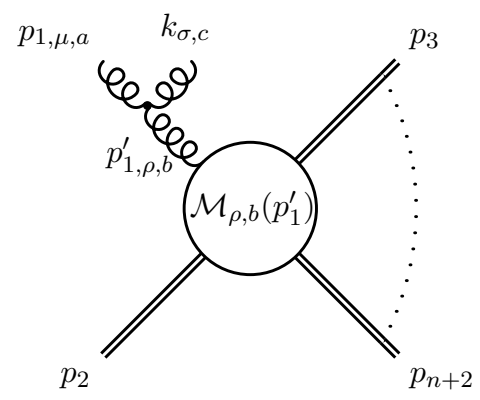

(c)

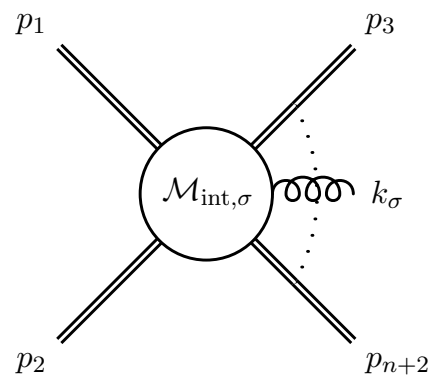

(f)

Figure 1. (a) Feynman diagram for a generic $2 \rightarrow n$ scattering process where all external particles are colour charged and either in the adjoint, fundamental or anti-fundamental representation, which are all indicated by a double line. By $\mathcal{M}_{\mathrm{H}}$ we denote the matrix element without any additional radiation, containing all asymptotic states (spinors and polarisation vectors). (b) Feynman diagram with the emission of one additional gluon carrying momentum $k$ and colour $c$ from an initial state quark carrying momentum $p_{1}$ and colour $c_{i}$. The notation is such that $\mathcal{M}$ contains all colour generators, spinors and/or polarisation vectors, except for the ones stemming from the line that is emitting. Since the gluon carries away momentum $k$ and changes the colour of the quark line, this matrix element will depend on $p_{1}^{\prime}=p_{1}-k$ and carries a colour label $c_{j}$. (c) Feynman diagram with the emission of one additional gluon from an initial state gluon. (d) Feynman diagram with an emission off a final state quark. Here the hard scattering matrix element depends on $p_{i}^{\prime}=p_{i}+k$. (e) Feynman diagram with an emission off a final state gluon. (f) Feynman diagram where the gluon is emitted from an internal line. The matrix element $\mathcal{M}_{\text {int, } \sigma}$ contains all colour generators and external states, except for $\epsilon_{\sigma}^{*}(k)$.

The notation is such that $\mathcal{M}_{c_{j}}$ contains all colour generators, spinors and/or polarisation vectors, except for the ones stemming from the line that is emitting. We have also introduced the generator of Lorentz transformations for fermionic fields:

$$
S^{\sigma \mu} \equiv \frac{i}{4}\left[\gamma^{\sigma}, \gamma^{\mu}\right]
$$

In the second line of eq. (2.1), the first two terms in the bracket come from rewriting the Dirac propagator in a suitable form. The derivative term stems from Taylor expanding the hard scattering matrix element $\mathcal{M}_{c_{j}}\left(p_{1}-k\right)$ to first order in $k$, where we have assumed this to be Taylor expandable. This assumption will fail at higher loop orders due to the presence of virtual collinear singularities, as first studied by ref. [27], and further developed by subsequent works $[42-46,48,65]$. However, the assumption is valid for tree- and loop- 
induced Born processes that are free of virtual collinear effects, which applies to all examples studied in this paper. Carrying out a similar exercise for the case where the initial state is an antiquark, we find

$$
\begin{aligned}
i \mathcal{M}_{1, \bar{q}}= & \frac{i g_{s} t_{c_{i} c_{j}}^{c}}{\left(p_{1}-k\right)^{2}+i \varepsilon} \bar{v}\left(p_{1}\right)\left(\left(2 p_{1}^{\sigma}-k^{\sigma}\right)+2 i S^{\sigma \mu} k_{\mu}-2 p_{1}^{\sigma} k^{\mu} \frac{\partial}{\partial p_{1}^{\mu}}\right) \\
& \times \mathcal{M}_{c_{j}}\left(p_{1}, p_{2}, \ldots, p_{n+2}\right) \epsilon_{\sigma}^{*}(k) .
\end{aligned}
$$

In the case of an emitting gluon (figure 1c) the resulting matrix element is:

$$
\begin{aligned}
i \mathcal{M}_{1, g}= & \frac{g_{s} f^{c a b}}{\left(p_{1}-k\right)^{2}+i \varepsilon} \epsilon_{\mu}\left(p_{1}\right) \mathcal{M}_{\rho, b}\left(p_{1}-k, p_{2}, \ldots, p_{n+2}\right)\left(-g^{\sigma \mu}\left(p_{1}+k\right)^{\rho}+g^{\mu \rho}\left(2 p_{1}-k\right)^{\sigma}\right. \\
& \left.+2 g^{\sigma \rho} k^{\mu}\right) \epsilon_{\sigma}^{*}(k) \\
= & \frac{g_{s} f^{c a b}}{\left(p_{1}-k\right)^{2}+i \varepsilon} \epsilon_{\mu}\left(p_{1}\right)\left(\left(2 p_{1}-k\right)^{\sigma} g^{\rho \mu}-2 i k_{\alpha} M^{\sigma \alpha, \rho \mu}-2 p_{1}^{\sigma} k^{\alpha} \frac{\partial}{\partial p_{1}^{\alpha}} g^{\rho \mu}\right) \\
& \times \mathcal{M}_{\rho, b}\left(p_{1}, p_{2}, \ldots, p_{n+2}\right) \epsilon_{\sigma}^{*}(k),
\end{aligned}
$$

where

$$
M^{\sigma \alpha, \rho \mu}=i\left(g^{\sigma \rho} g^{\mu \alpha}-g^{\sigma \mu} g^{\rho \alpha}\right)
$$

denotes the Lorentz generator for spin 1 particles and $\mathcal{M}_{\rho, b}$ the hard scattering matrix element with adjoint colour index $b$ and Minkowski index $\rho$ for the incoming gluon. To go from the first to the second line in eq. (2.4) we have used the physical polarisation condition for the incoming gluon to write [65]

$$
p_{1}^{\rho} \mathcal{M}_{\rho}\left(p_{1}, p_{2}, \ldots, p_{n+2}\right) \equiv 0 \rightarrow p_{1}^{\rho} k^{\alpha} \frac{\partial}{\partial p_{1}^{\alpha}} \mathcal{M}_{\rho}\left(p_{1}, p_{2}, \ldots, p_{n+2}\right)=-k^{\rho} \mathcal{M}_{\rho}\left(p_{1}, p_{2}, \ldots, p_{n+2}\right)
$$

We thus observe that for all species of incoming parton, the next-to-soft matrix element with an additional gluon emission from the incoming leg consists of three terms: a universal scalar term (which is proportional to $2 p_{i}^{\sigma}-k^{\sigma}$ ), a term that is sensitive to the spin of the emitter (which is proportional to either $S$ or $M$ ) and a universal derivative term acting on the nonradiative amplitude.

We may carry out a similar analysis for hard emitting particles in the final state. However, the fact that the gluon is emitted after the hard scattering results in a sign difference for the derivative term. More specifically, in considering the emission of a gluon of momentum $k$ from a final state hard particle of momentum $p_{i}+k$ leads to a momentumshifted amplitude

$$
\begin{aligned}
\mathcal{M}\left(p_{1}, p_{2}, \ldots, p_{i}+k, \ldots, p_{n+2}\right)= & \mathcal{M}\left(p_{1}, p_{2}, \ldots, p_{i}, \ldots, p_{n+2}\right) \\
& +k^{\alpha} \frac{\partial}{\partial p_{i}^{\alpha}} \mathcal{M}\left(p_{1}, p_{2}, \ldots, p_{i}, \ldots, p_{n+2}\right) .
\end{aligned}
$$

The next-to-soft matrix element for a final state quark emitter (figure 1d) is then found to be

$$
\begin{aligned}
i \mathcal{M}_{i, q}= & -\frac{i g_{s} t_{c_{i} c_{j}}^{c}}{\left(p_{i}+k\right)^{2}+i \varepsilon} \bar{u}\left(p_{i}\right)\left(2 p_{i}^{\sigma}+k^{\sigma}+2 i S^{\alpha \sigma} k_{\alpha}+2 p_{i}^{\sigma} k^{\alpha} \frac{\partial}{\partial p_{i}^{\alpha}}\right) \\
& \otimes \mathcal{M}_{c_{j}}\left(p_{1}, p_{2}, \ldots, p_{n+2}\right) \epsilon_{\sigma}^{*}(k),
\end{aligned}
$$


and for a final state antiquark emitter:

$$
i \mathcal{M}_{i, \bar{q}}=\frac{i g_{s} t_{c_{j} c_{i}}^{c}}{\left(p_{i}+k\right)^{2}+i \varepsilon} \mathcal{M}_{c_{j}}\left(p_{1}, p_{2}, \ldots, p_{n+2}\right)\left(2 p_{i}^{\sigma}+k^{\sigma}-2 i S^{\alpha \sigma} k_{\alpha}+2 k^{\alpha} \frac{\overleftarrow{\partial}}{\partial p_{i}^{\alpha}} p_{i}^{\sigma}\right) v\left(p_{i}\right) \epsilon_{\sigma}^{*}(k)
$$

For a final state gluon emitter the next-to-soft matrix element is (figure 1e):

$$
\begin{aligned}
i \mathcal{M}_{i, g}= & \frac{g_{s} f^{c b a}}{\left(p_{i}+k\right)^{2}+i \varepsilon} \epsilon_{\mu}^{*}\left(p_{i}\right)\left(g^{\mu \rho}\left(2 p_{i}^{\sigma}+k^{\sigma}\right)+2 i M^{\sigma \alpha, \rho \mu} k_{\alpha}+2 g^{\mu \rho} p_{i}^{\sigma} k^{\alpha} \frac{\partial}{\partial p_{i}^{\alpha}}\right) \\
& \times \mathcal{M}_{\rho, b}\left(p_{1}, p_{2}, \ldots, p_{n+2}\right) \epsilon_{\sigma}^{*}(k) .
\end{aligned}
$$

As for an initial state emitter, the NLP amplitude for a final state emitter also consists of a universal scalar term, a term that is sensitive to the spin of the emitter and a universal derivative term.

So far, we have considered only emissions from the external legs of the non-radiative amplitude. We must also consider the emission of a gluon from inside the hard interaction, as shown in figure 1f. To this end, we may consider the Ward identity for the emitted gluon, which takes the form

$$
i \mathcal{M}_{\mathrm{NLP}, \sigma} k^{\sigma}=\sum_{j=1}^{n+2} i \mathcal{M}_{j, \sigma} k^{\sigma}+i \mathcal{M}_{\mathrm{int}, \sigma} k^{\sigma}=0 \quad \rightarrow \quad i \mathcal{M}_{\mathrm{int}, \sigma} k^{\sigma}=-\sum_{j=1}^{n+2} i \mathcal{M}_{j, \sigma} k^{\sigma},
$$

where $\mathcal{M}_{j, \sigma}$ is the contribution to the total matrix element arising from gluon emission from an external line $j$, each consisting of a scalar, spin and derivative contribution as shown above. It is straightforwardly verified that the scalar and spin contributions vanish automatically upon contracting with $k^{\sigma}$, leaving only the derivative contribution, so that upon removing the gluon 4 -momentum from both sides one obtains ${ }^{3}$

$$
i \mathcal{M}_{\mathrm{int}, \sigma}=\sum_{j} \eta_{j} g_{s} \mathbf{T}_{j} \otimes \frac{\partial}{\partial p_{j}^{\sigma}}\left[i \mathcal{M}_{\mathrm{H}}\right],
$$

where $\eta_{j}=+1(-1)$ for a hard emitting particle in the initial (final) state respectively. We use the symbol $\otimes$ to denote the fact that the action of the colour generator for each external leg should be interpreted with appropriate coupling of colour indices to the hard interaction. The derivative does not act on the asymptotic states of the hard scattering matrix element $\mathcal{M}_{\mathrm{H}}$. Combining this expression with the other contributions above, we can now write down a general formula for the emission of a soft gluon from an arbitrary amplitude up to next-to-soft level:

$$
\begin{aligned}
\mathcal{A}_{\mathrm{NLP}} & =\mathcal{A}_{\mathrm{scal}}+\mathcal{A}_{\mathrm{spin}}+\mathcal{A}_{\mathrm{orb}} \\
& =\sum_{j=1}^{n+2} \frac{g_{s} \mathbf{T}_{j}}{2 p_{j} \cdot k}\left(\mathcal{O}_{\mathrm{scal}, j}^{\sigma}+\mathcal{O}_{\mathrm{spin}, j}^{\sigma}+\mathcal{O}_{\mathrm{orb}, j}^{\sigma}\right) \otimes i \mathcal{M}_{\mathrm{H}}\left(p_{1}, \ldots, p_{i}, \ldots, p_{n+2}\right) \epsilon_{\sigma}^{*}(k),
\end{aligned}
$$

\footnotetext{
${ }^{3}$ In principle, one may add a contribution $C_{\sigma}$ to the right-hand side of eq. (2.11), that is transverse by itself i.e. $k \cdot C=0$. Such contributions, however, can be ruled out based on gauge invariance and locality (see e.g. refs. [66, 67] for a recent discussion).
} 
where $\mathcal{M}_{\mathrm{H}}$ again denotes the hard scattering matrix element, and the first two terms on the right-hand side constitute the scalar-like and spin contributions respectively. Furthermore, the third term is the orbital angular momentum operator associated with each external leg, and $\mathbf{T}_{j}$ a colour generator in the appropriate representation. We use the symbol $\otimes$ in the same way as before, with the extension that now also the spin generator should be interpreted with the appropriate coupling of the spinor and/or vector indices to the hard interaction. We define each of these actions carefully, for all possible types of external leg, in appendix A. Note that the scalar contribution commences at LP in the soft expansion, whereas both of the angular momentum contributions are NLP only.

In eq. (2.13), the hard scattering matrix element contains only those momenta that are also present at LO. These do not obey momentum conservation once the extra radiation is present, and there appears to be an ambiguity in how one shares the momentum of the additional radiation between these existing momenta (see e.g. ref. [42] for a particularly complete discussion of this point). We will see in sections $3-5$ that actually there is no such ambiguity, as the form of the momentum shift created by the angular momentum operators of eq. (2.13) is completely fixed. Furthermore, exact momentum conservation is enforced by integrating over the complete phase space, which is not included in the amplitude itself.

The result of eq. (2.13) has previously been derived in a more formal context [40], where it is known as the next-to-soft theorem. It was motivated by a similar result in gravity [39, 41], that generalises the leading soft results of ref. [68]. Our reason for carefully rederiving this result here is twofold. Firstly, we may contrast this derivation with a similar analysis for the emission of soft quarks, to be carried out in the following section. Secondly, in applying eq. (2.13) to example scattering processes in the remainder of the paper, it is useful to have a precise record of how to keep track of colour and spinor/vector indices. The above derivation (and the results of appendix A) are particularly useful in this regard.

\subsection{Radiation of soft quarks}

Having reviewed the universal NLO amplitude for the emission of a (next-to-)soft gluon, we now turn to the emission of one additional soft quark. One must then consider all possible partonic splittings that can lead to such an emission, which we show for the case of emission from the initial state in figure 2. Let us first consider an initial state gluon splitting into a quark-antiquark pair, where the antiquark participates in the hard interaction (figure 2a). The resulting matrix element is

$$
i \mathcal{M}_{\mathcal{Q}, 1, g}=\frac{i g_{s} t_{c_{m} c_{j}}^{a}}{\left(p_{1}-k\right)^{2}+i \varepsilon} \epsilon^{\mu}\left(p_{1}\right) \bar{u}(k) \gamma_{\mu}(\not p /-\not k) \mathcal{M}_{c_{j}}\left(p_{1}-k, p_{2}, \ldots, p_{n+2}\right),
$$

where momenta and colour/Lorentz indices are labelled in the figure. The subscript $\mathcal{Q}$ is used to indicate the emission of a soft quark. From the fermion completeness relation for the emitted soft quark

$$
\sum_{\text {spins }} u(k) \bar{u}(k)=\not k,
$$

we see that the spinor for the emitted quark scales with soft momentum as $\mathcal{O}\left(k^{1 / 2}\right)$. Thus, the leading power of divergence for $k^{\sigma} \rightarrow 0$ in the matrix element is $\mathcal{O}\left(k^{-1 / 2}\right)$ (as opposed 


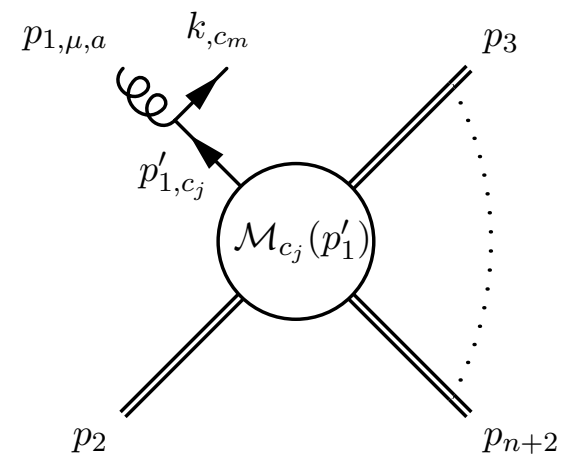

(a)

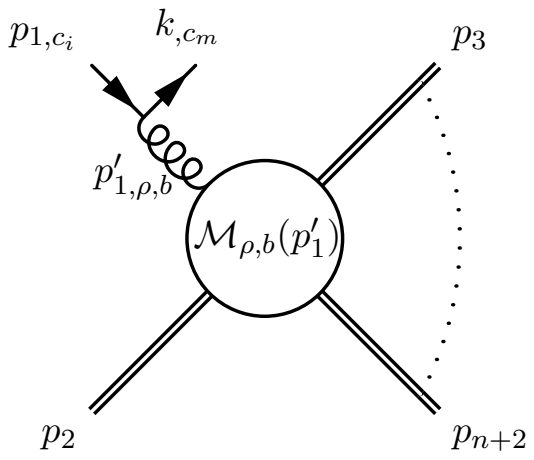

(b)

Figure 2. (a) Feynman diagram for the emission of one additional quark carrying momentum $k$ and colour $c_{m}$ from an initial state gluon carrying momentum $p_{1}$ and colour $a$. The momenta of the particles is defined to flow from left to right for all external lines. The hard scattering matrix element $\mathcal{M}_{c_{j}}\left(p_{1}^{\prime}\right)$ is defined to contain all external states, except for the polarisation vector $\epsilon^{\mu}\left(p_{1}\right)$ and the spinor $\bar{u}(k)$. By the emission of a quark, the identity and the colour of the external gluon changes. (b) Feynman diagram for the emission of one additional quark carrying momentum $k$ and colour $c_{m}$ from an initial state quark carrying momentum $p_{1}$ and colour $c_{i}$. The hard scattering matrix element $\mathcal{M}_{\rho, b}\left(p_{1}^{\prime}\right)$ is defined to contain all external states, except for the spinors $u\left(p_{1}\right)$ and $\bar{u}(k)$.

to $\mathcal{O}\left(k^{-1}\right)$ for the soft gluon case). It will therefore not give rise to a leading power threshold contribution, but will instead contribute at NLP accuracy. Furthermore, as the leading contribution from soft quark emission is already $\mathcal{O}\left(k^{-1 / 2}\right)$, there will be no additional contribution from the $\mathcal{O}(k)$ terms in the hard scattering matrix element or the Dirac propagator. The matrix element for soft quark emission becomes

$$
i \mathcal{M}_{\mathcal{Q}, 1, g}=\frac{i g_{s} t_{c_{m} c_{j}}^{a}}{\left(p_{1}-k\right)^{2}+i \varepsilon} \epsilon^{\mu}\left(p_{1}\right) \bar{u}(k) \gamma_{\mu} \not_{1} \mathcal{M}_{c_{j}}\left(p_{1}, p_{2}, \ldots, p_{n+2}\right) .
$$

A similar exercise can be performed if the initial state involves a quark splitting into a quark-gluon pair (figure 2b), and one obtains

$$
i \mathcal{M}_{\mathcal{Q}, 1, g}=\frac{i g_{s} t_{c_{m} c_{i}}^{b}}{\left(p_{1}-k\right)^{2}+i \varepsilon} \bar{u}(k) \gamma^{\rho} u\left(p_{1}\right) \mathcal{M}_{\rho, b}\left(p_{1}, p_{2}, \ldots, p_{n+2}\right) .
$$

The analysis for a final state particle emitting soft quarks is similar, as is the case of antiquark emission. Thus, we do not explicitly report the intermediate steps here. In the previous analysis of gluon emission, we needed to consider the possibility that a gluon was emitted from inside the hard scattering process, i.e. figure 1f. Here, while it is certainly possible that a soft quark is emitted from inside the hard scattering process, it will not give rise to an NLP contribution. This follows from the fact that the soft quark emission is by itself already a subleading effect in the momentum expansion, and also that any internal line is by definition far off-shell. Emission of an internal soft quark is then sub-sub-leading in the momentum expansion, thus irrelevant at NLP.

As for the gluon case, we can write a compact universal formula for soft quark emission. In order to do this, we introduce a quark emission operator $\mathcal{Q}_{i}$, which acts on a given external parton line $i$ to produce the emission of a quark or antiquark. The action of 

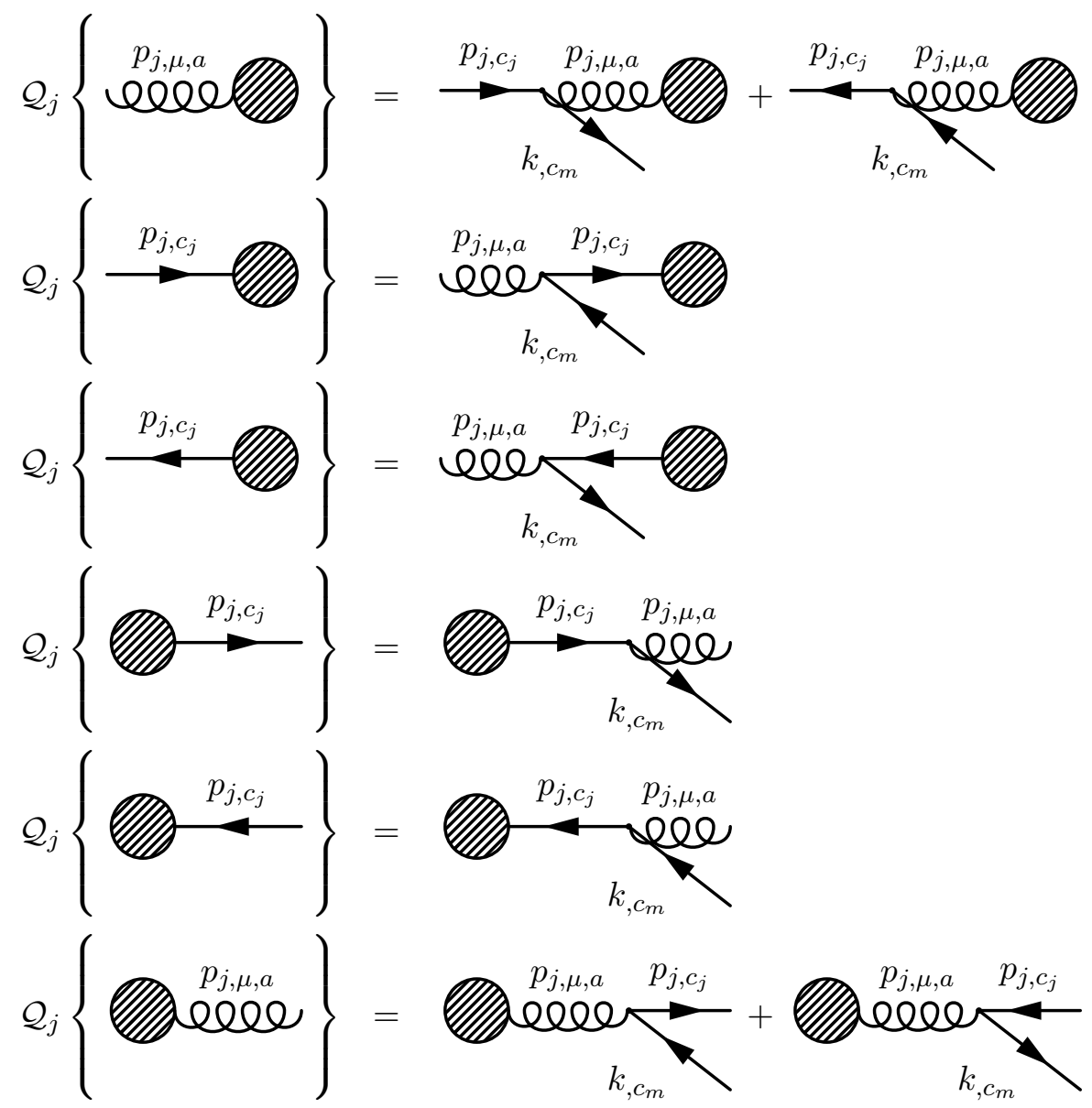

Figure 3. Action of the quark emission operator $\mathcal{Q}_{j}$ on an external parton line $j$, where all possible cases of incoming or outgoing line, and all parton species are considered. All momenta are defined to flow from left to right. The explicit contributions to the amplitude from each possibility are collected in appendix A.

this operator on every species of incoming/outgoing parton leg is shown diagrammatically in figure 3, and we collect the explicit rules for the amplitude from each possibility in appendix A. Armed with the quark emission operator, we may write the following general formula for the next-to-soft amplitude arising from soft (anti)quark emission:

$$
\mathcal{A}_{\mathrm{NLP}, \mathcal{Q}}=\sum_{j=1}^{n+2} \frac{g_{s}}{2 p_{j} \cdot k} \mathcal{Q}_{j} \otimes i \mathcal{M}_{H, j}\left(p_{1}, p_{2}, \ldots, p_{j}, \ldots, p_{n+2}\right) .
$$

This is very different to the next-to-soft gluon formalism of eq. (2.13), in that there is no equivalent of the scalar and orbital angular momentum contributions. The quark emission operator generates a single "external emission" from the non-radiative amplitude, that commences at NLP in the momentum expansion. Unlike the gluon case, it must change the identity of the parton that enters the hard scattering process.

In this and the previous section, we have derived a universal next-to-soft amplitude for the emission of a single additional gluon from a general Born process, up to next-to- 
soft order in its momentum. We have also derived a similar result for the emission of soft (anti)quarks, which involved introducing the quark emission operator of figure 3. As already noted above, the use of these formulae is more limited than in the case of processes without final state jets, considered in the previous work of ref. [60]. When final state jets are involved, the next-to-soft and threshold expansions no longer coincide, due to the presence of hard-collinear radiation. However, (next-to)-soft radiation is fully captured by the above results, so one expects that the leading-logarithmic terms at LP and NLP in the threshold expansion are correctly obtained. ${ }^{4}$ This may be illustrated by considering specific scattering processes, which we now turn to.

\section{$3 \quad$ NLP contributions in DIS at NLO}

In this section, we will consider arguably the simplest process that has an unobserved parton in the final state, namely deep inelastic scattering (DIS):

$$
e^{-}(k)+q(p) \rightarrow e^{-}\left(k^{\prime}\right)+q\left(k_{2}\right)
$$

We can then use the formulae derived in section 2.1 and 2.2 to compute the NLO crosssection, up to next-to-soft order in the momentum of the emitted radiation. For the calculation we will utilise dimensional regularisation in $d=4-2 \varepsilon$ dimensions, and use $\mu$ to indicate the renormalisation scale. The strong coupling is denoted as usual by $\alpha_{s} \equiv$ $\alpha_{s}\left(\mu^{2}\right)=g_{s}^{2} /(4 \pi)$. As is customary (see e.g. refs. [69, 70]), the complete squared amplitude before summing/averaging over spins, polarisations and colours can be written as

$$
|\mathcal{A}|^{2} \sim L^{\mu \nu} H_{\mu \nu}
$$

where $H^{\mu \nu}\left(L^{\mu \nu}\right)$ is the hadronic (leptonic) tensor respectively. To calculate the proton structure functions, it is sufficient to consider the hadronic tensor only. Thus, we may assume an initial state consisting of a quark and a spacelike off-shell photon, as shown in figure 4. Let us now consider the NLO cross-section up to NLP, which we calculate using eq. (2.13). For the hadronic tensor, we need the squared amplitude with different space-time indices for the off-shell photon, which reads

$$
\mathcal{A}^{\mu} \mathcal{A}^{\dagger \nu}=\mathcal{A}_{\mathrm{scal}}^{\mu} \mathcal{A}_{\mathrm{scal}}^{\dagger \nu}+2 \operatorname{Re}\left[\mathcal{A}_{\mathrm{scal}}^{\mu} \mathcal{A}_{\mathrm{spin}}^{\dagger \nu}+\mathcal{A}_{\mathrm{scal}}^{\mu} \mathcal{A}_{\mathrm{orb}}^{\dagger \nu}\right]+\ldots
$$

where we have included only those terms in the squared amplitude that are up to NLP in the next-to-soft expansion. By explicit calculation (similar to those performed in ref. [60]), we find that the first term on the right-hand side of eq. (3.2) is given by

$$
\left\langle\mathcal{A}_{\mathrm{scal}, \mu} \mathcal{A}_{\mathrm{scal}, \nu}^{\dagger}\right\rangle=g_{s}^{2} C_{F} \frac{p \cdot k_{2}}{(p \cdot k)\left(k_{2} \cdot k\right)} \operatorname{Tr}\left[k_{2} \mathcal{M}_{\mu}\left(p, k_{2}\right) \not p \mathcal{M}_{\nu}^{\dagger}\left(p, k_{2}\right)\right],
$$

where the bracket notation indicates that we have averaged over the initial state color and spin of the quark (resulting in a factor of $\frac{1}{2 N_{C}}$ ), and summed over final state spins and gluon

\footnotetext{
${ }^{4}$ Note that soft quark emission may lead to NLL terms at leading power, due to the fact that some of this soft radiation is collinear.
} 


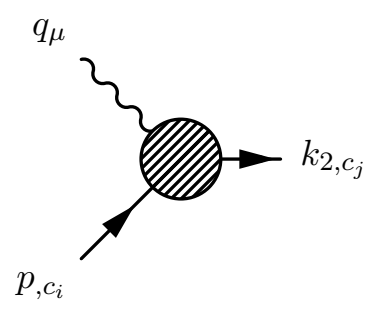

(a)

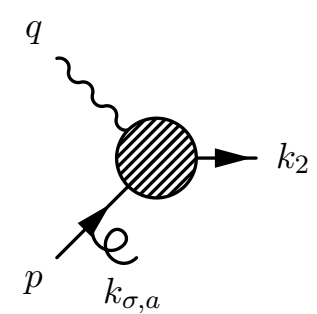

(b)

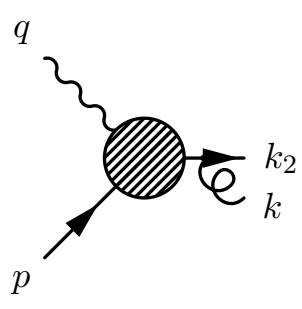

(c)

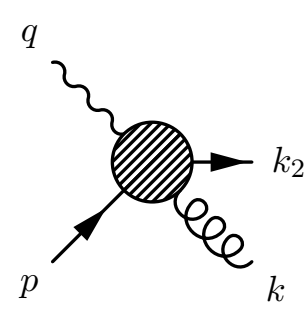

(d)

Figure 4. Diagrams for the DIS process: (a) shows the LO contribution, whilst (b)-(d) show all possible gluon emissions at NLO for the quark channel. Here $\left(p, q, k_{2}\right)$ denote the 4-momenta, $\mu$ and $\sigma$ are Lorentz indices, and $c_{i}(a)$ denotes a colour index in the fundamental (adjoint) representation.

polarisations. Furthermore, we have defined $\mathcal{M}^{\mu}\left(p, k_{2}\right) \equiv \mathcal{M}^{\mu}$ to be the LO amplitude, with external fermion wavefunctions removed, which we have allowed at present to be fully general. The scalar-spin contribution is found to be

$$
\left\langle 2 \operatorname{Re}\left[\mathcal{A}_{\mathrm{scal}, \mu} \mathcal{A}_{\mathrm{spin}, \nu}^{\dagger}\right]\right\rangle=-g_{s}^{2} C_{F}\left(\frac{1}{p \cdot k}-\frac{1}{k \cdot k_{2}}\right) \operatorname{Tr}\left[k_{2} \mathcal{M}_{\mu} \not p \mathcal{M}_{\nu}^{\dagger}\right] .
$$

Finally, the scalar-orbital squared amplitude is given by

$$
\begin{aligned}
\left\langle 2 \operatorname{Re}\left[\mathcal{A}_{\mathrm{scal}, \mu} \mathcal{A}_{\mathrm{orb}, \nu}^{\dagger}\right]\right\rangle=g_{s}^{2} C_{F} \frac{p \cdot k_{2}}{(p \cdot k)\left(k_{2} \cdot k\right)}[ & \operatorname{Tr}\left[k_{2} \mathcal{M}_{\mu} \not \phi\left(\delta p \cdot \frac{\partial}{\partial p}-\delta k_{2} \cdot \frac{\partial}{\delta k_{2}}\right) \mathcal{M}_{\nu}^{\dagger}\right] \\
& \left.+\operatorname{Tr}\left[\not p \mathcal{M}_{\nu}^{\dagger} k_{2}\left(\delta p \cdot \frac{\partial}{\partial p}-\delta k_{2} \cdot \frac{\partial}{\partial k_{2}}\right) \mathcal{M}_{\mu}\right]\right],
\end{aligned}
$$

where we have defined the momentum shifts

$$
\begin{aligned}
\delta p^{\alpha} & \equiv-\frac{1}{2}\left(k^{\alpha}+\frac{k_{2} \cdot k}{p \cdot k_{2}} p^{\alpha}-\frac{p \cdot k}{p \cdot k_{2}} k_{2}^{\alpha}\right) \\
\delta k_{2}^{\alpha} & \equiv-\frac{1}{2}\left(k^{\alpha}+\frac{p \cdot k}{p \cdot k_{2}} k_{2}^{\alpha}-\frac{k_{2} \cdot k}{p \cdot k_{2}} p^{\alpha}\right) .
\end{aligned}
$$

We are now in a position to calculate the full NLP squared amplitude for DIS. First we will make use of the chain rule to write

$$
\begin{aligned}
\left\langle 2 \operatorname{Re}\left[\mathcal{A}_{\mathrm{scal}, \mu} \mathcal{A}_{\mathrm{orb}, \nu}^{\dagger}\right]\right\rangle=g_{s}^{2} C_{F} \frac{p \cdot k_{2}}{(p \cdot k)\left(k_{2} \cdot k\right)}[ & \left(\delta p \cdot \frac{\partial}{\partial p}-\delta k_{2} \cdot \frac{\partial}{\partial k_{2}}\right) \operatorname{Tr}\left[k_{2} \mathcal{M}_{\mu} \not p \mathcal{M}_{\nu}^{\dagger}\right] \\
& \left.+\operatorname{Tr}\left[\delta k_{2} \mathcal{M}_{\mu} \not p \mathcal{M}_{\nu}^{\dagger}\right]-\operatorname{Tr}\left[k_{2} \mathcal{M}_{\mu} \delta / p \mathcal{M}_{\nu}^{\dagger}\right]\right] .
\end{aligned}
$$

The first term generates a momentum shift on the entire trace. The other two terms can be rewritten using a Sudakov decomposition for the emitted gluon momentum:

$$
k^{\mu}=\frac{p \cdot k}{p \cdot k_{2}} k_{2}^{\mu}+\frac{k_{2} \cdot k}{p \cdot k_{2}} p^{\mu}+k_{T}^{\mu},
$$


so that one finds

$$
\begin{aligned}
\left\langle 2 \operatorname{Re}\left[\mathcal{A}_{\mathrm{scal}, \mu} \mathcal{A}_{\mathrm{orb}, \nu}^{\dagger}\right]\right\rangle=g_{s}^{2} C_{F} \frac{p \cdot k_{2}}{(p \cdot k)\left(k_{2} \cdot k\right)}[ & \left(\delta p \cdot \frac{\partial}{\partial p}-\delta k_{2} \cdot \frac{\partial}{\partial k_{2}}\right) \operatorname{Tr}\left[k_{2} \mathcal{M}_{\mu} \not p \mathcal{M}_{\nu}^{\dagger}\right] \\
+ & \left.\frac{k_{2} \cdot k}{p \cdot k_{2}} \operatorname{Tr}\left[k_{2} \mathcal{M}_{\mu} \not p \mathcal{M}_{\nu}^{\dagger}\right]-\frac{p \cdot k}{p \cdot k_{2}} \operatorname{Tr}\left[k_{2} \mathcal{M}_{\mu} \not p \mathcal{M}_{\nu}^{\dagger}\right]\right] .
\end{aligned}
$$

Here we have ignored terms linear in $k_{T}$, as they will ultimately vanish upon integration over the final state phase space. The latter two terms can be combined with the scalar-spin contribution in eq. (3.4), after which they also vanish. Putting everything together, the complete NLP squared amplitude can be written in terms of the LO hadronic tensor

$$
H_{\mu \nu}\left(p, k_{2}\right)=\left\langle\mathcal{A}_{\mu}^{(0)}\left(p, k_{2}\right) \mathcal{A}_{\nu}^{(0))^{\dagger}}\left(p, k_{2}\right)\right\rangle
$$

but with momenta shifted according to eq. (3.7):

$$
\left.\left\langle\mathcal{A}_{\mu} \mathcal{A}_{\nu}^{\dagger}\right\rangle\right|_{\mathrm{LP}+\mathrm{NLP}}=g_{s}^{2} C_{F} \frac{p \cdot k_{2}}{(p \cdot k)\left(k_{2} \cdot k\right)} H_{\mu \nu}\left(p+\delta p, k_{2}-\delta k_{2}\right) .
$$

This is directly analogous to the case of colour singlet production examined in ref. [60], which also found that the squared amplitude for the one real emission contribution could be written in terms of the momentum-shifted non-radiative amplitude. The forms of the shifts found here differ only in that the shift in $k_{2}$ has an opposite sign, owing to the fact that it is a final-, rather than initial-state momentum. Up to now we have allowed the LO stripped amplitude to be fully general, but we now use the explicit result for DIS: ${ }^{5}$

$$
\mathcal{M}^{\mu}=\gamma^{\mu},
$$

before projecting the squared amplitude of eq. (3.12) with:

$$
T_{2}^{\mu \nu}=-\frac{1}{4 \pi} \frac{1}{2-2 \varepsilon}\left(g^{\mu \nu}+(3-2 \varepsilon) \frac{q^{2}}{(p \cdot q)^{2}} p^{\mu} p^{\nu}\right)
$$

to obtain the proton structure function $F_{2}^{\gamma}\left(x, Q^{2}\right)$ (see e.g. refs. $\left.[69,70]\right) .{ }^{6}$ To calculate the structure function, we use the following momentum parameterisation [69, 70]:

$$
\begin{aligned}
p & =\frac{s+Q^{2}}{2 \sqrt{s}}(1,0, \ldots, 0,1) \\
q & =\left(\frac{s-Q^{2}}{2 \sqrt{s}}, 0, \ldots, 0,-\frac{s+Q^{2}}{2 \sqrt{s}}\right) \\
k & =\frac{\sqrt{s}}{2}(1,0, \ldots, 0, \sin \theta, \cos \theta) \\
k_{2} & =\frac{\sqrt{s}}{2}(1,0, \ldots, 0,-\sin \theta,-\cos \theta) .
\end{aligned}
$$

\footnotetext{
${ }^{5}$ Note that we have not included a factor of $i Q_{q} g_{\mathrm{EM}}$ here, which we define to be part of the leptonic tensor.

${ }^{6}$ One may also consider the structure function $F_{L}$. However, this does not exhibit any logarithmic terms at NLO. See ref. [32] for a detailed discussion of threshold contributions at higher orders.
} 
Next defining $\cos \theta=2 y-1$ and $s=\frac{Q^{2}(1-x)}{x}$, one has

$$
-q^{2}=Q^{2}, \quad p \cdot k=\frac{Q^{2}(1-y)}{2 x}, \quad p \cdot q=\frac{Q^{2}}{2 x}, \quad q \cdot k=\frac{Q^{2}(y-x)}{2 x},
$$

such that the two-body final state phase space may be written as

$$
\int \mathrm{d} \Phi_{2}=\frac{2 \pi}{(4 \pi)^{d / 2} \Gamma\left(\frac{d-4}{2}\right)}\left(\frac{Q^{2}}{\mu^{2}}\right)^{d / 2-2}\left(\frac{1-x}{x}\right)^{\frac{d-2}{2}} \int_{0}^{1} \mathrm{~d} y(y(1-y))^{\frac{d-4}{2}} .
$$

Note that there is an overall factor of $x^{-(d-4) / 2} \equiv x^{\varepsilon}$, which when expanded about $x=1$ contributes to NLP terms in the final result suppressed by a power of $\varepsilon$. That is, this term contributes as $\varepsilon \ln (1-x)$. Therefore, corrections of the phase space will not affect leading logarithmic behaviour in this case. Using these ingredients, the result for the structure function, valid up to NLP, is

$$
\begin{aligned}
F_{2, \mathrm{LP}+\mathrm{NLP}}^{\gamma}\left(x, Q^{2}\right) & =\left.\int \mathrm{d} \Phi_{2} T_{2}^{\mu \nu}\left\langle\mathcal{A}_{\mu} \mathcal{A}_{\nu}^{\dagger}\right\rangle\right|_{\mathrm{LP}+\mathrm{NLP}} \\
& =\frac{\alpha_{s}}{4 \pi}\left(-\frac{4}{\varepsilon} \frac{1}{1-x}+\frac{4}{\varepsilon}-\frac{4-4 \ln (1-x)}{1-x}+8-4 \ln (1-x)+\mathcal{O}(1-x)\right),
\end{aligned}
$$

where we have set

$$
\bar{\mu}^{2} \equiv 4 \pi \mathrm{e}^{-\gamma_{E}} \mu^{2}=Q^{2} .
$$

This is the result obtained for the structure function in the next-to-soft approximation, as opposed to the full NLO result

$$
F_{2, \mathrm{NLO}}^{\gamma}\left(x, Q^{2}\right)=\frac{\alpha_{s}}{4 \pi}\left(-\frac{4}{\varepsilon} \frac{1}{1-x}+\frac{4}{\varepsilon}-\frac{3-4 \ln (1-x)}{1-x}+14-4 \ln (1-x)+\mathcal{O}(1-x)\right) .
$$

Comparison of eqs. (3.17) and (3.19) shows that the next-to-soft expansion indeed correctly captures the dominant threshold terms at both LP and NLP (aided by the above-noted absence of phase space corrections at leading logarithmic order). The discrepancy between the next-to-soft and full calculations up to NLP level is

$$
F_{2, \mathrm{NLO}}^{\gamma}\left(x, Q^{2}\right)-F_{2, \mathrm{LP}+\mathrm{NLP}}^{\gamma}\left(x, Q^{2}\right)=\frac{\alpha_{s}}{4 \pi}\left(\frac{1}{1-x}+6+\mathcal{O}(1-x)\right) .
$$

This consists of a LP term which, when combined with virtual corrections, will yield a subleading (NLL) plus-distribution. As is well-known, this can be captured by introducing a jet function [3], which includes the hard-collinear effects that are missing in the next-tosoft expansion. We do not do so here, given that our aim is to classify which NLP effects are captured by using the next-to-soft expansion alone. Given that the NLP term in the discrepancy of eq. (3.20) is a numerical constant, we see that all LL terms at LP and NLP are correctly captured by the next-to-soft formalism.

Our soft-quark framework allows us to include the other partonic DIS channel in a natural way, namely the one where the hard scattering is induced by a gluon that splits 


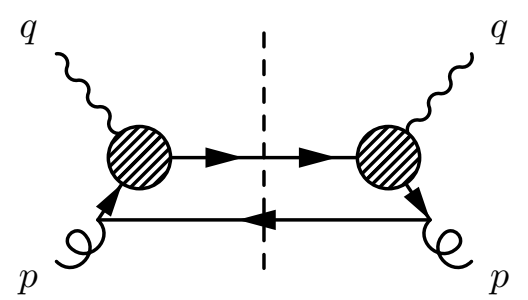

Figure 5. Contributions for the DIS $F_{2, q}\left(x, Q^{2}\right)$ structure function, where the (anti)quark is soft.

into a quark-antiquark pair, shown for convenience in figure 5. This contribution turns out to be

$$
F_{2, q}^{\gamma}=\frac{\alpha_{s}}{4 \pi}\left(-\frac{2}{\varepsilon}+2 \ln (1-x)+\mathcal{O}(1-x)\right) .
$$

One observes the presence of a pole associated with the initial state quark-antiquark pair becoming collinear (but such that the quark is still next-to-soft). This in turn generates an NLP logarithmic term in the finite part, due to the interplay of the collinear pole with the factor $(1-x)^{-\varepsilon}$ in the 2-body phase space of eq. (3.16).

In this section, we have examined a first process with a final state parton (DIS), and found that the next-to-soft formalism as derived in section 2.1 can be used to derive a similar result to that obtained for colour singlet particle production in ref. [60]. That is, LL LP and NLP threshold contributions in the NLO amplitude are captured in terms of the LO amplitude, with shifted external momenta (eq. (3.12)). Contrary to ref. [60], only the LL terms are captured, as expected given that final state collinear enhancements are present at the first subleading logarithmic order. However, the momentum shift formula remains a very useful result for practical applications, given that it correctly predicts all LL terms at NLP level. To investigate how general this situation is, it is instructive to consider a second inclusive process with two final state jets, which we do in the following section.

\section{NLP contributions in $e^{+} e^{-} \rightarrow$ jets at NLO}

The next process we will consider is that of hadroproduction in electron-positron annihilation:

$$
e^{+}\left(p_{1}\right)+e^{-}\left(p_{2}\right) \rightarrow \gamma(q) \rightarrow q\left(k_{1}\right)+\bar{q}\left(k_{2}\right)+g(k),
$$

where we will choose $q\left(k_{1}\right)$ to fragment into the observed hadron, and be inclusive for the other final state particles. We are interested in the transverse parton fragmentation function (see e.g. [71] $)^{7}$

$$
\mathcal{F}_{T}\left(z, Q^{2}\right)=\frac{1}{d-2}\left(-\frac{2 k_{1} \cdot q}{q^{2}} W_{\mu}^{\mu}-\frac{2}{k_{1} \cdot q} k_{1}^{\mu} k_{2}^{\nu} W_{\mu \nu}\right) .
$$

Here $W_{\mu \nu}$ is the parton structure tensor

$$
W_{\mu \nu}(p, q)=\frac{z^{d-3}}{4 \pi} \int \mathrm{d} \Phi_{2}\left\langle\mathcal{A}_{\mu} \mathcal{A}_{\nu}^{\dagger}\right\rangle,
$$

\footnotetext{
${ }^{7}$ We could also consider the longitudinal fragmentation function. However, as for the longitudinal structure function $F_{L}$ in DIS, this does not contain logarithmic contributions at NLO.
} 
where $\mathcal{A}_{\mu}$ now denotes the matrix element of the sub-process $\gamma^{*}(q) \rightarrow q\left(k_{1}\right) \bar{q}\left(k_{2}\right) g(k)$, and we have also defined the partonic scaling variable

$$
z=\frac{2 k_{1} \cdot q}{Q^{2}}
$$

The phase space for the two unobserved final state partons takes the form

$$
\int \mathrm{d} \Phi_{2}=(\mu)^{4-d} \int \frac{\mathrm{d}^{d} k}{(2 \pi)^{d-1}} \delta^{+}\left(k^{2}\right) \frac{\mathrm{d}^{d} k_{2}}{(2 \pi)^{d-1}} \delta^{+}\left(k_{2}^{2}\right)(2 \pi)^{d} \delta^{(d)}\left(q-k_{1}-k_{2}-k\right),
$$

where the momenta of the photon and the outgoing partons can be parameterised as follows [71]:

$$
\begin{aligned}
q & =\sqrt{s}(1,0, \ldots, 0) \\
k_{1} & =\frac{s-s_{12}}{2 \sqrt{s}}(1,0, \ldots, 0,1) \\
k_{2} & =\frac{s-s_{2}}{2 \sqrt{s}}(1,0, \ldots, 0, \sin \theta, \cos \theta) \\
k & =q-k_{1}-k_{2} .
\end{aligned}
$$

We have introduced the invariants

$$
s=Q^{2}, \quad s_{1}=\left(k_{1}+k_{2}\right)^{2}, \quad s_{2}=\left(k_{1}+k\right)^{2}, \quad s_{12}=\left(k_{2}+k\right)^{2},
$$

satisfying $s=s_{1}+s_{2}+s_{12}$. Using momentum conservation and the on-shell conditions for the antiquark and the gluon, we can parameterise the phase space in a convenient way using

$$
\cos \theta=\frac{s_{2} s_{12}-s_{1} s}{\left(s-s_{12}\right)\left(s-s_{2}\right)}, \quad s_{1}=z(1-y) s, \quad s_{12}=(1-z) s, \quad s_{2}=y z s,
$$

such that the 2-body phase space reads

$$
\int \mathrm{d} \Phi_{2}=\frac{1}{8 \pi} \frac{1}{\Gamma(1-\varepsilon)}\left(\frac{4 \pi \mu^{2}}{s}\right)^{\varepsilon}(1-z)^{-\varepsilon} \int_{0}^{1} \mathrm{~d} y(y(1-y))^{-\varepsilon} .
$$

As in the case of DIS, we may now calculate the one real emission correction to the $\gamma^{*} \rightarrow$ $q+X$ amplitude up to NLP, by applying the next-to-soft formalism of eq. (2.13). This proceeds directly analogously to the previous calculation, and we find that the squared amplitude may be written as

$$
\left.\left\langle\mathcal{A}_{\mu} \mathcal{A}_{\nu}^{\dagger}\right\rangle\right|_{\mathrm{LP}+\mathrm{NLP}}=g_{s}^{2} C_{F} \frac{k_{1} \cdot k_{2}}{\left(k_{1} \cdot k\right)\left(k_{2} \cdot k\right)} H_{\mu \nu}^{\gamma^{*} \rightarrow q \bar{q}}\left(k_{1}-\delta k_{1}, k_{2}-\delta k_{2}\right),
$$

where the squared Born process is denoted by $H_{\mu \nu}^{\gamma^{*} \rightarrow q \bar{q}}$ and the momentum shifts are defined via

$$
\begin{aligned}
\delta k_{1}^{\alpha} & =-\frac{1}{2}\left(k^{\alpha}+\frac{k_{2} \cdot k}{k_{1} \cdot k_{2}} k_{1}^{\alpha}-\frac{k_{1} \cdot k}{k_{1} \cdot k_{2}} k_{2}^{\alpha}\right) \\
\delta k_{2}^{\alpha} & =-\frac{1}{2}\left(k^{\alpha}+\frac{k_{1} \cdot k}{k_{1} \cdot k_{2}} k_{2}^{\alpha}-\frac{k_{2} \cdot k}{k_{1} \cdot k_{2}} k_{1}^{\alpha}\right) .
\end{aligned}
$$


Thus, as in the DIS and colour singlet production cases [60], we again find that we can describe the squared soft-gluon amplitude using a momentum shift of the non-radiative amplitude. The momentum shifts are similar in form to the previous cases, but both have a negative sign owing to the fact that both hard partons are now in the final state. Putting together all of the above ingredients, the next-to-soft result for the parton fragmentation function $\mathcal{F}_{T, \mathrm{LP}+\mathrm{NLP}}\left(z, Q^{2}\right)$ is ${ }^{8}$

$$
\begin{aligned}
\mathcal{F}_{T, \mathrm{LP}+\mathrm{NLP}}\left(z, Q^{2}\right)=\frac{\alpha_{s} z}{\pi}\left(\frac{1}{\varepsilon} \frac{1}{1-z}-\frac{1}{\varepsilon}-\frac{\ln (1-z)}{1-z}+\ln (1-z)\right. \\
\left.\quad-\frac{z}{1-z}\left(2 \ln z-1+\ln \frac{s}{\bar{\mu}^{2}}\right)\right) .
\end{aligned}
$$

This may be compared with the full NLO expression, which is

$$
\begin{aligned}
\mathcal{F}_{T, \mathrm{NLO}}\left(z, Q^{2}\right)=\frac{\alpha_{s} z}{4 \pi} & \left(\frac{2}{\varepsilon} \frac{1+z^{2}}{1-z}-2 \frac{\ln (1-z)}{1-z}\left(1+z^{2}\right)\right. \\
& \left.-\frac{1}{1-z}\left(4\left(1+z^{2}\right) \ln z+3(z-2) z+2\left(1+z^{2}\right) \ln \frac{s}{\bar{\mu}^{2}}\right)\right) .
\end{aligned}
$$

Similar to the DIS case, the next-to-soft formalism predicts the LL behaviour, but fails to capture a LP term and a constant piece at NLP:

$$
\begin{aligned}
\mathcal{F}_{T, \mathrm{NLO}}\left(z, Q^{2}\right)-\mathcal{F}_{T, \mathrm{LP}+\mathrm{NLP}}\left(z, Q^{2}\right) & = \\
& \frac{\alpha_{s} z}{4 \pi}\left(-\frac{1}{1-z}+4+(1-z)\left[\frac{2}{\varepsilon}-3-2 \ln (1-z)-2 \ln \frac{s}{\bar{\mu}^{2}}-4 \ln z\right]\right) .
\end{aligned}
$$

The LP term would be described by a conventional jet function. The missing NLP term is again a numerical constant, so we conclude that the LL LP and NLP terms are correctly captured by the next-to-soft formalism.

In this section we have examined a second example of a scattering process containing a final state parton. We again find that we can successfully use eq. (2.13) to obtain an NLO result for the amplitude that captures all LL threshold contributions up to NLP.

\section{NLP cross-section for NLO prompt photon production}

In this section we will consider the production of a single photon that recoils against a hard parton at NLO, where the latter is unobserved. This process has more partonic subchannels than the ones previously considered and it has more than one colour structure to consider at NLO. This makes the prompt photon production process an interesting testing ground for our next-to-soft gluon and soft quark formalisms. Furthermore, it is not straightforward to determine where the different NLP threshold contributions originate from. This section thus aims to disentangle the NLP contributions, and show their origin.

\footnotetext{
${ }^{8}$ Following eq. (4.2), we keep an overall factor of $z$ unexpanded, which would cancel with a similar factor in forming the hadronic fragmentation function.
} 

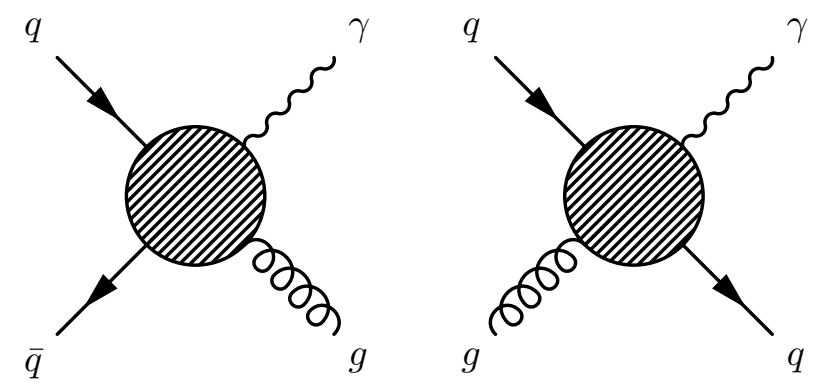

Figure 6. Feynman diagrams for the LO processes $q \bar{q} \rightarrow g \gamma$ (left) and $q g \rightarrow \gamma q$ (right).

At leading order, the prompt photon production process $(p p \rightarrow \gamma+X)$ consists of two subprocesses: $q \bar{q} \rightarrow \gamma g$ and $q g \rightarrow q \gamma$, as shown in figure 6. At next-to-leading order in the coupling, one additional particle can be radiated. This creates a variety of new diagrams that one has to consider. Firstly, one can add an additional gluon to the two Born processes: $q \bar{q} \rightarrow \gamma g g$ and $q g \rightarrow q g \gamma$. The presence of this additional gluon will create the leading power logarithmic contributions to the NLO cross-section. Apart from that, there will be diagrams that appear for the first time at NLO and contain an extra quark in the final state: $q \bar{q} \rightarrow \gamma q^{\left({ }^{\prime}\right)} \bar{q}^{\left({ }^{\prime}\right)}, g g \rightarrow \gamma q \bar{q}$ and $q q^{\left({ }^{\prime}\right)} \rightarrow \gamma q q^{\left({ }^{\prime}\right)}$. Although only the two Born processes need to be considered for a leading power analysis, all of the other additional subprocesses will also contribute at NLP only due to the possibility of a final state unobserved quark becoming soft.

Due to the presence of different subprocesses, we have divided this section into three subsections. In subsection 5.1 we will consider the channel that only has gluons in the final state: $q \bar{q} \rightarrow g g \gamma$. Then, in subsection 5.2, we consider the subchannel $q \bar{q} \rightarrow q \bar{q} \gamma$, which does not contribute at LL LP and only commences at LL NLP. In the last subsection, we will consider the most involved example, namely one where both gluons and quarks are present in the final state. We will find there that the next-to-soft gluon effects are factorised from the soft quark effects, therefore their contributions can be calculated separately, as was suggested in section 2. For our calculations we will use $\mu_{F}$ to indicate the factorisation scale and the electromagnetic fine structure constant is given by $\alpha=g_{\mathrm{EM}}^{2} /(4 \pi)$.

\section{$5.1 \quad q \bar{q} \rightarrow g g \gamma$ channel}

We first consider the process obtained by dressing $q \bar{q} \rightarrow g \gamma$ with one additional gluon, where the relevant Feynman diagrams for this are shown in figure 7. Given that gluons are the only partons in the final state, it should be sufficient to describe this process, up to next-to-soft level, with the amplitude of eq. (2.13). To compute the cross-section, we must calculate the squared amplitude, summed and averaged over final/initial state colours and spins. This involves summing over all polarisations for the emitted gluon, and one may restrict this to be over physical degrees of freedom by defining an arbitrary lightlike vector $l$ such that $l \cdot \epsilon(k)=0$, where $\epsilon_{\mu}(k)$ is the polarisation vector of the gluon, thus obtaining

$$
\sum_{\text {phys. }} \epsilon_{\alpha}^{\dagger}(k) \epsilon_{\beta}(k)=-\eta_{\alpha \beta}+\frac{l_{\alpha} k_{\beta}+l_{\beta} k_{\alpha}}{l \cdot k} .
$$


Alternatively, one may sum over all polarisations (including an unphysical longitudinal degree of freedom), provided one introduces external ghost particles to remove the spurious contributions. As argued in ref. [60], however, soft ghosts do not contribute up to NLP in the momentum expansion, and thus one may replace eq. (5.1) with ${ }^{9}$

$$
\sum_{\text {pols. }} \epsilon_{\alpha}^{\dagger}(k) \epsilon_{\beta}(k)=-\eta_{\alpha \beta}
$$

Since the current process is more involved than the DIS and $e^{+} e^{-}$cases due to the presence of more than two NLO diagrams, we will again provide explicit details of how to construct the next-to-soft squared amplitude, thus illustrating the use of eq. (2.13) in a more complicated scenario. As before, we will start with the scalar amplitude

$$
\begin{aligned}
\mathcal{A}_{\text {scal }}=\bar{v}\left(p_{2}\right) & \left(\frac{i g_{s} t_{c_{k} c_{i}}^{b}\left(2 p_{1}^{\sigma}-k^{\sigma}\right)}{2 p_{1} \cdot k} \mathcal{M}_{c_{j} c_{k}}^{\mu \nu, a}-\frac{i g_{s} t_{c_{j} c_{k}}^{b}\left(2 p_{2}^{\sigma}-k^{\sigma}\right)}{2 p_{2} \cdot k} \mathcal{M}_{c_{k} c_{i}}^{\mu \nu, a}\right. \\
\left.-\frac{g_{s} f^{b a c}\left(2 p_{R}^{\sigma}+k^{\sigma}\right)}{2 p_{R} \cdot k} g_{\rho}^{\mu} \mathcal{M}_{c_{j} c_{i}}^{\rho \nu, c}\right) u\left(p_{1}\right) \epsilon_{\mu}^{*}\left(p_{R}\right) \epsilon_{\nu}^{*}\left(p_{\gamma}\right) \epsilon_{\sigma}^{*}(k) &
\end{aligned}
$$

where the matrices $\mathcal{M}_{c_{j} c_{k}}^{\mu \nu, a}, \mathcal{M}_{c_{k} c_{i}}^{\mu \nu, a}$ and $\mathcal{M}_{c_{j} c_{i}}^{\rho \nu, c}$ correspond to the shaded circles in the first three diagrams in figure 7 , whose dependence on the momenta $p_{1}, p_{2}, p_{R}$ and $p_{\gamma}$ is implicitly understood. For clarity we have included explicit colour labels on the matrix element, indicating that it still depends on the colour structure via the $\mathrm{SU}(3)$ generators. The double scalar contribution to the matrix element squared, inclusive of spin/colour averaging factors, is easily computed and results in

$$
\begin{aligned}
& \left\langle\left|\mathcal{A}_{\text {scal }}\right|^{2}\right\rangle=\frac{Q_{q}^{2} g_{\mathrm{EM}}^{2} g_{s}^{4} C_{F}}{4 C_{A}} \operatorname{Tr}\left[\not p_{2} \Gamma^{\mu \nu} \not p_{1} \Gamma_{\mu \nu}^{*}\right] \times\left[C_{F} \frac{2 p_{1} \cdot p_{2}}{\left(p_{1} \cdot k\right)\left(p_{2} \cdot k\right)}\right. \\
& \left.+\frac{1}{2} C_{A}\left(\frac{2 p_{1} \cdot p_{R}}{\left(p_{1} \cdot k\right)\left(p_{R} \cdot k\right)}+\frac{2 p_{2} \cdot p_{R}}{\left(p_{2} \cdot k\right)\left(p_{R} \cdot k\right)}-\frac{2 p_{1} \cdot p_{2}}{\left(p_{1} \cdot k\right)\left(p_{2} \cdot k\right)}\right)\right] .
\end{aligned}
$$

Here $\Gamma^{\mu \nu} \equiv \Gamma^{\mu \nu}\left(p_{1}, p_{2}, p_{R}, p_{\gamma}\right)$ denotes the (non-radiative) hard scattering matrix element for the process $q\left(p_{1}\right) \bar{q}\left(p_{2}\right) \rightarrow g\left(p_{R}\right) \gamma\left(p_{\gamma}\right)$ stripped of its polarisation vectors, spinors, colour factors and charges, and where we suppress the momenta labels for brevity.

We now move on to the scalar-spin interference term. The spin amplitude for the $q \bar{q} \rightarrow g g \gamma$ process evaluates to

$$
\begin{aligned}
\mathcal{A}_{\text {spin }}= & \bar{v}\left(p_{2}\right)\left(\frac{i g_{s} t_{c_{k} c_{i}}^{b}}{2 p_{1} \cdot k} \mathcal{M}_{c_{j} c_{k}}^{\mu \nu, a} \gamma^{\sigma} \not k-\frac{i g_{s} t_{c_{j} c_{k}}^{b}}{2 p_{2} \cdot k} \not k \gamma^{\sigma} \mathcal{M}_{c_{k} c_{i}}^{\mu \nu, a}+\frac{g_{s} f^{b a c}}{p_{R} \cdot k} \mathcal{M}_{c_{j} c_{i}}^{\rho \nu, c}\left(g_{\rho}^{\sigma} k^{\mu}-g^{\sigma \mu} k_{\rho}\right)\right) \\
& \times u\left(p_{1}\right) \epsilon_{\mu}^{*}\left(p_{R}\right) \epsilon_{\nu}^{*}\left(p_{\gamma}\right) \epsilon_{\sigma}^{*}(k) .
\end{aligned}
$$

Contracting the spin amplitude with the scalar amplitude then results in

$$
2 \operatorname{Re}\left[\mathcal{A}_{\text {scal }} \mathcal{A}_{\text {spin }}^{*}\right]=-\frac{Q_{q}^{2} g_{\mathrm{EM}}^{2} g_{s}^{4} C_{F}^{2}}{4 C_{A}} \frac{2 p_{1} \cdot p_{2}}{\left(p_{1} \cdot k\right)\left(p_{2} \cdot k\right)} \frac{\left(p_{1}+p_{2}\right) \cdot k}{p_{1} \cdot p_{2}} \operatorname{Tr}\left[\not p_{2} \Gamma^{\mu \nu} \not p_{1} \Gamma_{\mu \nu}^{*}\right]
$$

\footnotetext{
${ }^{9} \mathrm{~A}$ similar distinction was not needed for the DIS and $e^{+} e^{-}$processes, due to the fact that ghosts cannot couple directly to quarks.
} 

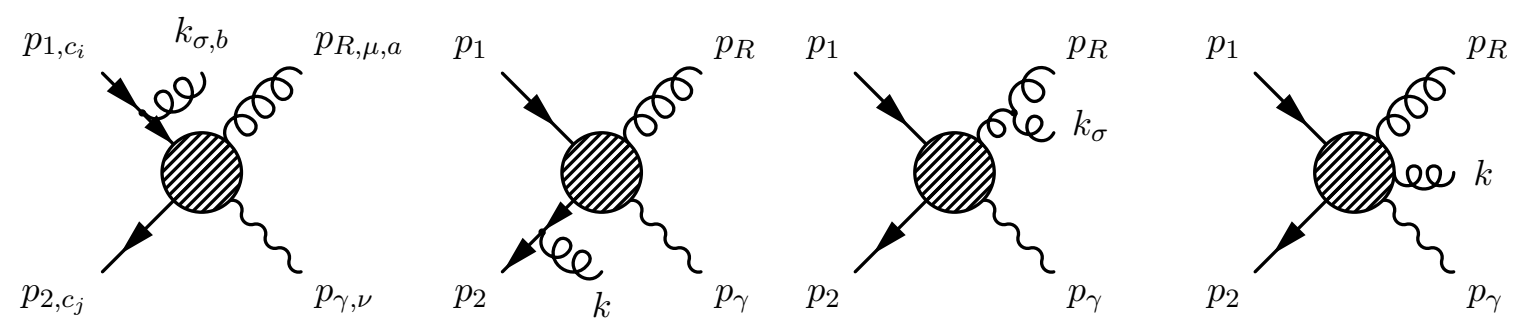

Figure 7. NLO Feynman diagrams for the NLP contributions of the process $q\left(p_{1}\right) \bar{q}\left(p_{2}\right) \rightarrow$ $g\left(p_{R}\right) g(k) \gamma\left(p_{\gamma}\right)$.

Finally, we must evaluate the scalar-orbital interference term. To this end, we may first write down the orbital amplitude

$$
\begin{aligned}
\mathcal{A}_{\text {orb }}=i g_{s} k_{\alpha} \bar{v}\left(p_{2}\right) & \frac{t_{c_{k} c_{i}}^{b}}{p_{1} \cdot k}\left(p_{1}^{\alpha} \frac{\partial}{\partial p_{1, \sigma}}-p_{1}^{\sigma} \frac{\partial}{\partial p_{1, \alpha}}\right) \mathcal{M}_{c_{j} c_{k}}^{\mu \nu, a}-\frac{t_{c_{j} c_{k}}^{b}}{p_{2} \cdot k}\left(p_{2}^{\alpha} \frac{\partial}{\partial p_{2, \sigma}}-p_{2}^{\sigma} \frac{\partial}{\partial p_{2, \alpha}}\right) \mathcal{M}_{c_{k} c_{i}}^{\mu \nu, a} \\
& \left.-\frac{i f^{b a c}}{p_{R} \cdot k}\left(p_{R}^{\alpha} \frac{\partial}{\partial p_{R, \sigma}}-p_{R}^{\sigma} \frac{\partial}{\partial p_{R, \alpha}}\right) \mathcal{M}_{c_{j} c_{i}}^{\mu \nu, c}\right) u\left(p_{1}\right) \epsilon_{\mu}^{*}\left(p_{R}\right) \epsilon_{\nu}^{*}\left(p_{\gamma}\right) \epsilon_{\sigma}^{*}(k),
\end{aligned}
$$

to be contracted with the scalar amplitude. Adopting the abbreviation

$$
\delta p_{i ; j}^{\alpha} \equiv-\frac{1}{2}\left(k^{\alpha}+\frac{p_{j} \cdot k}{p_{i} \cdot p_{j}} p_{i}^{\alpha}-\frac{p_{i} \cdot k}{p_{i} \cdot p_{j}} p_{j}^{\alpha}\right)
$$

we get

$$
\begin{aligned}
\left\langle\mathcal{A}_{\mathrm{orb}} \mathcal{A}_{\mathrm{scal}}^{*}\right\rangle= & \frac{Q_{q}^{2} g_{\mathrm{EM}}^{2} g_{s}^{4} C_{F}}{4 C_{A}}\left[\left(C_{F}-\right.\right. \\
& \left.\frac{1}{2} C_{A}\right) \frac{2 p_{1} \cdot p_{2}}{\left(p_{1} \cdot k\right)\left(p_{2} \cdot k\right)} \\
& \times \operatorname{Tr}\left[\not p_{2}\left(\delta p_{1 ; 2}^{\alpha} \frac{\partial}{\partial p_{1}^{\alpha}}+\delta p_{2 ; 1}^{\alpha} \frac{\partial}{\partial p_{2}^{\alpha}}\right) \Gamma^{\mu \nu} \not p_{1} \Gamma_{\mu \nu}^{*}\right] \\
& +\frac{1}{2} C_{A} \frac{2 p_{1} \cdot p_{R}}{\left(p_{1} \cdot k\right)\left(p_{R} \cdot k\right)} \operatorname{Tr}\left[\not p_{2}\left(\delta p_{1 ; R}^{\alpha} \frac{\partial}{\partial p_{1}^{\alpha}}-\delta p_{R ; 1}^{\alpha} \frac{\partial}{\partial p_{R}^{\alpha}}\right) \Gamma^{\mu \nu} \not p_{1} \Gamma_{\mu \nu}^{*}\right] \\
& \left.+\frac{1}{2} C_{A} \frac{2 p_{2} \cdot p_{R}}{\left(p_{2} \cdot k\right)\left(p_{R} \cdot k\right)} \operatorname{Tr}\left[\not p_{2}\left(\delta p_{2 ; R}^{\alpha} \frac{\partial}{\partial p_{2}^{\alpha}}-\delta p_{R ; 2}^{\alpha} \frac{\partial}{\partial p_{R}^{\alpha}}\right) \Gamma^{\mu \nu} \not p_{1} \Gamma_{\mu \nu}^{*}\right]\right] .
\end{aligned}
$$

The expression for $\left\langle\mathcal{A}_{\text {scal }} \mathcal{A}_{\text {orb }}^{*}\right\rangle$ looks similar, but with the derivatives acting on $\Gamma_{\mu \nu}^{*}$. As in section 3 , we may transform the derivatives in these expressions into total derivatives acting on the complete trace using the chain rule, which results in

$$
\begin{aligned}
\left\langle 2 \operatorname{Re}\left[\mathcal{A}_{\mathrm{orb}} \mathcal{A}_{\mathrm{scal}}^{*}\right]\right\rangle= & \frac{Q_{q}^{2} g_{\mathrm{EM}}^{2} g_{s}^{4} C_{F}}{4 C_{A}}\left[C _ { F } \frac { 2 p _ { 1 } \cdot p _ { 2 } } { ( p _ { 1 } \cdot k ) ( p _ { 2 } \cdot k ) } \left\{\left(\delta p_{1 ; 2}^{\alpha} \frac{\partial}{\partial p_{1}^{\alpha}}+\delta p_{2 ; 1}^{\alpha} \frac{\partial}{\partial p_{2}^{\alpha}}\right) \operatorname{Tr}\left[\not p_{2} \Gamma^{\mu \nu} \not p_{1} \Gamma_{\mu \nu}^{*}\right]\right.\right. \\
& \left.-\operatorname{Tr}\left[\delta \not p_{2 ; 1} \Gamma^{\mu \nu} \not p_{1} \Gamma_{\mu \nu}^{*}\right]-\operatorname{Tr}\left[\not p_{2} \Gamma^{\mu \nu} \delta \not p_{1 ; 2} \Gamma_{\mu \nu}^{*}\right]\right\} \\
& +\frac{1}{2} C_{A} \frac{2 p_{1} \cdot p_{R}}{\left(p_{1} \cdot k\right)\left(p_{R} \cdot k\right)}\left(\delta p_{1 ; R}^{\alpha} \frac{\partial}{\partial p_{1}^{\alpha}}-\delta p_{R ; 1}^{\alpha} \frac{\partial}{\partial p_{R}^{\alpha}}\right) \operatorname{Tr}\left[\not p_{2} \Gamma^{\mu \nu} \not p_{1} \Gamma_{\mu \nu}^{*}\right] \\
& +\frac{1}{2} C_{A} \frac{2 p_{2} \cdot p_{R}}{\left(p_{2} \cdot k\right)\left(p_{R} \cdot k\right)}\left(\delta p_{2 ; R}^{\alpha} \frac{\partial}{\partial p_{2}^{\alpha}}-\delta p_{R ; 2}^{\alpha} \frac{\partial}{\partial p_{R}^{\alpha}}\right) \operatorname{Tr}\left[\not p_{2} \Gamma^{\mu \nu} \not p_{1} \Gamma_{\mu \nu}^{*}\right] \\
& \left.-\frac{1}{2} C_{A} \frac{2 p_{1} \cdot p_{2}}{\left(p_{1} \cdot k\right)\left(p_{2} \cdot k\right)}\left(\delta p_{1 ; 2}^{\alpha} \frac{\partial}{\partial p_{1}^{\alpha}}+\delta p_{2 ; 1}^{\alpha} \frac{\partial}{\partial p_{2}^{\alpha}}\right) \operatorname{Tr}\left[\not p_{2} \Gamma^{\mu \nu} \not p_{1} \Gamma_{\mu \nu}^{*}\right]\right] .
\end{aligned}
$$


Here we see explicitly the presence of two different colour structures, namely terms in the square bracket which are proportional to $C_{F}$ and $C_{A}$ respectively. For the $C_{F}$ contribution, the derivative term is accompanied by additional contributions, involving the shifts $\delta \not p_{2 ; 1}$ and $\delta \not p_{1 ; 2}$. A similar situation occurred in eq. (3.8) for the DIS process, where the additional contributions ended up being cancelled by the spin-scalar interference term. The same turns out to happen here if one introduces a Sudakov decomposition for $k$, defined here such that

$$
\delta p_{i ; j}^{\alpha}=-\frac{1}{2}\left(k_{T(i, j)}^{\alpha}+2 \frac{p_{j} \cdot k}{p_{i} \cdot p_{j}} p_{i}^{\alpha}\right)
$$

where $k_{T(i, j)}$ is defined to be orthogonal to both $p_{i}$ and $p_{j}$. As in the DIS and $e^{+} e^{-}$case, terms that are proportional to $k_{T}$ will ultimately vanish upon integration over the final state phase space, so we will ignore them in what follows. Interestingly, for the $C_{A}$ term there is no need for a cancellation originating from a spin-scalar contribution, as the terms proportional to $C_{A}$ vanish directly up to $\mathcal{O}\left(k_{T}\right)$. Putting all pieces together, the complete LP + NLP squared amplitude can then be written as

$$
\begin{aligned}
\left\langle\left|\mathcal{A}_{\mathrm{LP}+\mathrm{NLP}, q \bar{q} \rightarrow \gamma g g}\right|^{2}\right\rangle=\frac{Q_{q}^{2} g_{\mathrm{EM}}^{2} g_{s}^{4} C_{F}}{4} C_{A} & {\left[\left(C_{F}-\frac{1}{2} C_{A}\right) \frac{2 p_{1} \cdot p_{2}}{\left(p_{1} \cdot k\right)\left(p_{2} \cdot k\right)}\right.} \\
\times & H_{q \bar{q} \rightarrow \gamma g}\left(p_{1}+\delta p_{1 ; 2}, p_{2}+\delta p_{2 ; 1}, p_{\gamma}, p_{R}\right) \\
& +\frac{1}{2} C_{A} \frac{2 p_{1} \cdot p_{R}}{\left(p_{1} \cdot k\right)\left(p_{R} \cdot k\right)} H_{q \bar{q} \rightarrow \gamma g}\left(p_{1}+\delta p_{1 ; R}, p_{2}, p_{\gamma}, p_{R}-\delta p_{R ; 1}\right) \\
& \left.+\frac{1}{2} C_{A} \frac{2 p_{2} \cdot p_{R}}{\left(p_{2} \cdot k\right)\left(p_{R} \cdot k\right)} H_{q \bar{q} \rightarrow \gamma g}\left(p_{1}, p_{2}+\delta p_{2 ; R}, p_{\gamma}, p_{R}-\delta p_{R ; 2}\right)\right],
\end{aligned}
$$

where $H_{q \bar{q} \rightarrow \gamma g}\left(p_{1}+\delta p_{1 ; 2}, p_{2}+\delta p_{2 ; 1}, p_{\gamma}, p_{R}\right)$ denotes the trace appearing in e.g. eq. (5.10), but where the momenta $p_{1}$ and $p_{2}$ are shifted by $\delta p_{1 ; 2}$ and $\delta p_{2 ; 1}$ respectively. This result is directly analogous to the previous cases, which also found that the squared amplitude for the one real emission contribution could be written in terms of the momentum-shifted non-radiative amplitude. There is a notable difference with respect to our previous results, however. Both the DIS and $e^{+} e^{-}$cases had only two parton legs in the LO process, and the final result for the squared amplitude consisted of a dipole-like eikonal factor dressing the momentum shifted hard interaction (eqs. (3.12) and (4.6) respectively). In the present case, we see multiple dipole-like terms, each consisting of an eikonal factor involving two hard momenta $p_{i}$ and $p_{j}$ dressing a hard interaction where the same momenta are shifted. Furthermore, different dipole terms have correspondingly different colour structures.

As remarked above, the momentum shifts in eq. (5.12) are generated by a combination of the spin and orbital term for the $C_{F}$ colour structure and only by the orbital term for the $C_{A}$ colour structure. The orbital terms act as a momentum shift operator on the hard scattering of the matrix element, while the spin term takes care of the same shift on the asymptotic states.

We are now in a position to integrate over the final state momenta $p_{R}$ and $k$ and compute the differential cross-section. We will separate the three-body phase space into two two-body phase spaces, one containing the unobserved partons, the other describing 
the photon and the collective effect of the unobserved partons. To this end, we first factor the three-body phase space as

$$
\mathrm{d} \Phi_{3}=\frac{1}{2 \pi} \int \mathrm{d} P^{2} \mathrm{~d} \Phi_{2}\left(p_{1}+p_{2} ; p_{\gamma}, P\right) \mathrm{d} \Phi_{2}\left(P ; p_{R}, k\right),
$$

where the second two-body phase space factor on the right-hand side is in the center of mass frame of the two unobserved partons, and takes the form

$$
\mathrm{d} \Phi_{2}\left(P ; p_{R}, k\right)=\left(\frac{16 \pi \mu^{2}}{P^{2}}\right)^{\varepsilon} \frac{1}{16 \pi \Gamma\left(\frac{1}{2}\right) \Gamma\left(\frac{1}{2}-\varepsilon\right)} \int_{0}^{\pi} \mathrm{d} \theta_{1}\left(\sin \theta_{1}\right)^{1-2 \varepsilon} \int_{0}^{\pi} \mathrm{d} \theta_{2}\left(\sin \theta_{2}\right)^{-2 \varepsilon}
$$

after having parameterised the momenta as follows:

$$
\begin{aligned}
p_{R} & =\frac{\sqrt{P^{2}}}{2}\left(1,0, \ldots, 0, \sin \theta_{1} \sin \theta_{2}, \sin \theta_{1} \cos \theta_{2}, \cos \theta_{1}\right) \\
k & =\frac{\sqrt{P^{2}}}{2}\left(1,0, \ldots, 0,-\sin \theta_{1} \sin \theta_{2},-\sin \theta_{1} \cos \theta_{2},-\cos \theta_{1}\right) .
\end{aligned}
$$

In terms of the invariants $s=\left(p_{1}+p_{2}\right)^{2}, u_{1}=\left(p_{1}-p_{\gamma}\right)^{2}, t_{1}=\left(p_{2}-p_{\gamma}\right)^{2}$ and $s_{4}=$ $\left(p_{1}+p_{2}-p_{\gamma}\right)^{2}=\left(k+p_{R}\right)^{2}$, the other phase space is given by

$$
\mathrm{d} \Phi_{2}\left(p_{1}+p_{2} ; p_{\gamma}, P\right)=\left(\frac{4 \pi s \mu^{2}}{t_{1} u_{1}}\right)^{\varepsilon} \frac{1}{8 \pi s \Gamma(1-\varepsilon)} \delta^{+}\left(P^{2}-s_{4}\right) \int \mathrm{d} t_{1} \int \mathrm{d} u_{1} .
$$

To compare our results with the NLO calculation presented in ref. [72], we will make a change of variables:

$$
\begin{aligned}
& u_{1} \equiv-s v w \\
& t_{1} \equiv s(v-1) \\
& s_{4}=s+t_{1}+u_{1}=s v(1-w),
\end{aligned}
$$

where $(1-w)$ plays the role of the threshold variable $\xi$ in eq. (1.1) (i.e. $w \rightarrow 1$ at threshold). In terms of these invariants the complete three body phase space now reads

$$
\mathrm{d} \Phi_{3}=s\left(\frac{4 \pi \mu^{2}}{s}\right)^{2 \varepsilon} \frac{v\left(v^{2}(1-v) w(1-w)\right)^{-\varepsilon}}{(4 \pi)^{4} \Gamma(1-2 \varepsilon)} \int \mathrm{d} v \int \mathrm{d} w \int_{0}^{\pi} \mathrm{d} \theta_{1}\left(\sin \theta_{1}\right)^{1-2 \varepsilon} \int_{0}^{\pi} \mathrm{d} \theta_{2}\left(\sin \theta_{2}\right)^{-2 \varepsilon} .
$$

Furthermore, we will extract a common factor of $v w(1-v) s$ from the differential crosssection as was done in ref. [72], and we obtain

$$
\begin{aligned}
v w(1-v) s \frac{\mathrm{d} \sigma_{q \bar{q} \rightarrow \gamma g g}^{\mathrm{LP}+\mathrm{NLP}}}{\mathrm{d} v \mathrm{~d} w}= & s\left(\frac{4 \pi \mu^{2}}{s}\right)^{2 \varepsilon} \frac{v^{2} w(1-v)\left(v^{2}(1-v) w(1-w)\right)^{-\varepsilon}}{2(4 \pi)^{4} \Gamma(1-2 \varepsilon)} \\
& \int_{0}^{\pi} \mathrm{d} \theta_{1}\left(\sin \theta_{1}\right)^{1-2 \varepsilon} \int_{0}^{\pi} \mathrm{d} \theta_{2}\left(\sin \theta_{2}\right)^{-2 \varepsilon}\left\langle\left|\mathcal{A}_{\mathrm{LP}+\mathrm{NLP}, q \bar{q} \rightarrow \gamma g g}\right|^{2}\right\rangle .
\end{aligned}
$$


We can now use our NLP result for the amplitude (eq. (5.12)). Inserting the form of the momentum shifts of eq. (5.8) and integrating, one finds

$$
\begin{aligned}
v w(1-v) s \frac{\mathrm{d} \sigma_{q \bar{q} \rightarrow \gamma g g}^{\mathrm{LP}+\mathrm{NLP}}}{\mathrm{d} v \mathrm{~d} w}= & Q_{q}^{2} \alpha \alpha_{s}^{2} \frac{C_{F}}{C_{A}}\left[-\frac{1}{\varepsilon} \frac{4 C_{F} T_{q \bar{q}}}{(1-w)_{+}}-\frac{1}{\varepsilon}\left\{2 C_{F} \frac{4 v(v-1)^{2}-1}{1-v}\right\}\right. \\
& +\left(\frac{\ln (1-w)}{1-w}\right)_{+} 2\left(4 C_{F}-C_{A}\right) T_{q \bar{q}} \\
& +\frac{1}{(1-w)_{+}}\left\{T_{q \bar{q}}\left(2 C_{A} \ln (1-v)+8 C_{F} \ln (v)-2 C_{A}-8 C_{F} \ln \frac{\bar{\mu}^{2}}{s}\right)\right. \\
& \left.+8 C_{F}((v-1) v+1)\right\} \\
& +\ln (1-w)\left\{\left(4 C_{F}-C_{A}\right) \frac{4 v(v-1)^{2}-1}{1-v}\right\} \\
& +\mathcal{O}(\delta(1-w))+\mathcal{O}(1)]
\end{aligned}
$$

where $T_{q \bar{q}}=2 v(v-1)+1$. We may compare this result with the full NLO calculation of ref. [72], collected for convenience up to NLP in the threshold expansion in appendix C. Upon doing so we observe that, as before, the next-to-soft formalism correctly reproduces LL terms at both LP and NLP. We also capture some of the NLL terms, but to complete these would again require taking into account hard-collinear information via the addition of a gluon jet function. However, it is reassuring that LL information is correctly reproduced even in a less inclusive situation.

The above cross-section contains infrared poles, that must be absorbed into the parton distributions via the usual mass factorisation procedure. This leads to a novel source of NLL NLP contributions in the fully subtracted cross-section. Although the focus of this paper is not to fully account for the NLL NLP contributions, we feel that it is worth drawing attention to this particular point, as this might be relevant for future numerical studies of NLP effects. Mass factorisation can be performed by adding a counter cross section, which is a convolution of a scaled Born cross section with the parton distribution functions. The phase space for the counter term consists of a two-body final state, and is given by

$$
\mathrm{d} \Phi_{2}=\left(\frac{4 \pi \mu^{2}}{s}\right)^{\varepsilon} \frac{(v(1-v))^{-\varepsilon}}{8 \pi \Gamma(1-\varepsilon)} \int \mathrm{d} v \int \mathrm{d} w \delta(1-w) .
$$

There is then a difference in the $\varepsilon$-dependence with respect to the three-body phase space of eq. (5.17), such that subtracting the counterterm leads schematically to an NLP contribution in the finite part of the cross-section:

$$
\begin{gathered}
\frac{1}{\varepsilon} \frac{1}{(1-w)_{+}}\left[\left(\frac{4 \pi \mu^{2}}{s}\right)^{2 \varepsilon} \frac{\left(v^{2}(1-v) w(1-w)\right)^{-\varepsilon}}{\Gamma(1-2 \varepsilon)}-\left(\frac{4 \pi \mu^{2}}{s}\right)^{\varepsilon} \frac{(v(1-v))^{-\varepsilon}}{\Gamma(1-\varepsilon)}\right] \\
=-\left(\frac{\ln (1-w)}{1-w}\right)_{+}+\frac{\ln \left(\bar{\mu}^{2} / s\right)}{(1-w)_{+}}-\frac{\ln (v)}{(1-w)_{+}}-\frac{\ln (w)}{(1-w)_{+}}
\end{gathered}
$$


The first and second term on the second line of this equation are part of the LL and NLL LP contributions, whereas the fourth term is of NLP, but not LL. The third term on the second line gives subleading logarithmic contributions (NLL) at both LP and NLP. This is confusing at first, but has everything to do with how the threshold for this one-particleinclusive process is defined. Traditionally, the threshold limit is chosen to be $4 p_{T}^{2} \rightarrow s$ [73], where $p_{T}$ is the transverse momentum of the observed photon. To perform threshold resummation, one needs to go to a conjugate space to perform the kinematic factorisation. The Mellin moment is taken with respect to $x_{T}^{2}=4 p_{T}^{2} / s$ and reads [73]

$$
\tilde{\sigma}(N)=\int_{0}^{1} \mathrm{~d} x_{T}^{2}\left(x_{T}^{2}\right)^{N-1} \frac{p_{T}^{3} \mathrm{~d} \sigma\left(p_{T}\right)}{\mathrm{d} p_{T}}=\frac{1}{2} \int_{0}^{1} \mathrm{~d} v \int_{0}^{1} \mathrm{~d} w(4 v(1-v) w)^{N+1} \frac{s \mathrm{~d} \sigma(v, w)}{\mathrm{d} v \mathrm{~d} w}
$$

where

$$
\int_{0}^{1} \mathrm{~d} v(4 v(1-v))^{N+1} f(v)=f\left(\frac{1}{2}\right)+\mathcal{O}\left(\frac{1}{N}\right) .
$$

The LP contributions are therefore fixed at $v=1 / 2$, with $\mathcal{O}(1 / N)$ terms appearing for $v \neq 1 / 2$. Such an $\mathcal{O}(1 / N)$ term originates in $(v, w)$-space from an NLL LP contribution, but in Mellin space it resembles an LL NLP contribution, since

$$
\begin{aligned}
& \int_{0}^{1} \mathrm{~d} w w^{N}\left(\frac{\ln (1-w)}{1-w}\right)_{+} \simeq \frac{1}{2}\left(\ln ^{2} \bar{N}+\frac{\ln \bar{N}}{N}\right)+\mathcal{O}(1) \\
& \int_{0}^{1} \mathrm{~d} w w^{N} \frac{1}{(1-w)_{+}} \simeq-\ln \bar{N}+\mathcal{O}\left(\frac{1}{N}\right)
\end{aligned}
$$

Here, the first term in eq. (5.24) is of LL LP, whereas the second term is of LL NLP. The term in eq. (5.25) is of NLL LP. However, the $\mathcal{O}(1 / N)$ term created by the Mellin transform in eq. (5.23) multiplied with the $\ln (N)$ contribution here creates a term that is of LL NLP in Mellin space. This could be a crucial ingredient in future numerical studies of NLP effects.

In this section we calculated the NLP contribution of the $q \bar{q} \rightarrow \gamma g g$ channel to the exclusive prompt photon production process. Similar to the DIS and $e^{+} e^{-}$processes, the next-to-soft gluon formalism indeed correctly reproduces the LL terms at both the LP and NLP. Having understood this particular partonic process, let us now consider a second subchannel in the following section.

\section{$5.2 \quad q \bar{q} \rightarrow q \bar{q} \gamma$ channel}

We now turn to the partonic sub-process of figure 8, whose final state contains only quarks in addition to the photon. To compute the NLP differential cross-section, we therefore only need to consider eq. (2.18). There are 5 types of contributions, indicated in figure 8 , which can be split into three categories: initial state splitting $(I)$, final state fragmentation $(F F)$ and final state splitting $(F)$. The contributions can be obtained by letting the quark emission operator $\mathcal{Q}_{j}$ act on the tree level processes $q g \rightarrow q \gamma, \bar{q} g \rightarrow \bar{q} \gamma, q \bar{q} \rightarrow g \gamma$ and $q \bar{q} \rightarrow q \bar{q}$. From the processes thus obtained, we then select only those with the specific 
partonic assignment $q \bar{q} \rightarrow q \bar{q} \gamma$. We obtain

$$
\begin{aligned}
& \mathcal{A}_{\mathrm{NLP}, \mathrm{quarks}}=\left[-\frac{i g_{s} t_{c_{j} c_{i}}^{a}}{2 p_{1} \cdot p_{R}} \bar{u}\left(p_{R}\right) \gamma_{\mu} u\left(p_{1}\right) \mathcal{M}_{(g) \bar{q} \rightarrow \bar{q} \gamma}^{\mu, a}\left(p_{1}, p_{2}, k, p_{\gamma}\right)\right. \\
&-\frac{\left.i g_{s} t_{c_{k} c_{m}}^{a} \bar{v}\left(p_{2}\right) \gamma_{\mu} v(k) \mathcal{M}_{q(g) \rightarrow q \gamma}^{\mu, a}\left(p_{1}, p_{2}, p_{R}, p_{\gamma}\right)\right]}{2 p_{2} \cdot k} \\
&+\frac{i g_{s} t_{c_{j} c_{m}}^{a}}{2 p_{R} \cdot k} \bar{u}\left(p_{R}\right) \gamma_{\mu} v(k) \mathcal{M}_{q \bar{q} \rightarrow(g) \gamma}^{\mu, a}\left(p_{1}, p_{2}, p_{R}, p_{\gamma}\right) \\
&+\left[-\frac{i g_{\mathrm{EM}} Q_{q}}{2 p_{\gamma} \cdot p_{R}} \bar{u}\left(p_{R}\right) \notin^{*}\left(p_{\gamma}\right) \not p_{\gamma} \mathcal{M}_{q \bar{q} \rightarrow(q) \bar{q}}\left(p_{1}, p_{2}, p_{\gamma}, k\right)\right. \\
&\left.+\frac{i g_{\mathrm{EM}} Q_{q}}{2 p_{\gamma} \cdot k} \mathcal{M}_{q \bar{q} \rightarrow q(\bar{q})}\left(p_{1}, p_{2}, p_{R}, p_{\gamma}\right) \not p_{\gamma} \notin^{*}\left(p_{\gamma}\right) v(k)\right] \\
& \equiv \mathcal{A}_{I}+ \mathcal{A}_{F F}+\mathcal{A}_{F},
\end{aligned}
$$

where the notation $(a)$ in each hard scattering matrix element $\mathcal{M}$ indicates that the latter does not include the external wavefunction for parton $a .{ }^{10}$ The complete NLP cross-section can be written as a sum of these contributions as

$$
v w(1-v) s \frac{\mathrm{d} \sigma_{q \bar{q} \rightarrow q \bar{q} \gamma}^{\mathrm{NLP}}}{\mathrm{d} v \mathrm{~d} w}=Q_{q}^{2} \alpha \alpha_{s}^{2}\left[\Sigma_{I, I}+\Sigma_{I, F}+\Sigma_{I, F F}+\Sigma_{F, F}+\Sigma_{F, F F}+\Sigma_{F F, F F}\right],
$$

where $\Sigma_{I, J}(I, J \in\{I, F, F F\})$ denotes the contribution from the integrated, summed and averaged soft quark squared amplitude $\left\langle\mathcal{A}_{I} \mathcal{A}_{J}^{\dagger}\right\rangle$ (plus the complex conjugate if $I \neq J$ ). The individual contributions are found to be

$$
\begin{aligned}
& \Sigma_{I, I}=-\frac{C_{F}}{2 C_{A}} \frac{1}{\varepsilon} \frac{2 v^{4}-4 v^{3}+4 v^{2}-2 v+1}{1-v}+\frac{C_{F}}{2 C_{A}} \ln (1-w) \frac{2 v^{4}-4 v^{3}+4 v^{2}-2 v+1}{1-v}+\mathcal{O}(1) \\
& \Sigma_{I, F}= \ln (1-w)\left\{\frac{C_{F}}{C_{A}^{2}} v^{2}(1-v)-\frac{C_{F}}{C_{A}} v(3-2 v(1-v))\right\}+\mathcal{O}(1) \\
& \Sigma_{I, F F}= \mathcal{O}(1) \\
& \Sigma_{F, F}=-\frac{1}{\varepsilon}\left\{\frac{C_{F}}{C_{A}^{2}} \frac{v\left(3 v^{3}-6 v^{2}+4 v-1\right)}{1-v}+\frac{C_{F}}{C_{A}} \frac{2 v^{6}-6 v^{5}+8 v^{4}-6 v^{3}+5 v^{2}-3 v+1}{1-v}\right\} \\
&+\ln (1-w)\left\{\frac{C_{F}}{C_{A}^{2}} \frac{v\left(3 v^{3}-6 v^{2}+4 v-1\right)}{1-v}\right\}+\mathcal{O}(1) \\
&\left.+\frac{C_{F}}{C_{A}} \frac{2 v^{6}-6 v^{5}+8 v^{4}-6 v^{3}+5 v^{2}-3 v+1}{1-v}\right\}
\end{aligned}
$$

$$
\begin{aligned}
\Sigma_{F, F F} & =\mathcal{O}(1) \\
\Sigma_{F F, F F} & =\frac{1}{(1-w)_{+}} \frac{C_{F}}{3 C_{A}} T_{q \bar{q}}+\mathcal{O}(1) .
\end{aligned}
$$

\footnotetext{
${ }^{10}$ In the third line of eq. (5.26), we have been careful to include only one part of the result of the $\mathcal{Q}$ operator so as to avoid double counting, as explained in appendix A.
} 


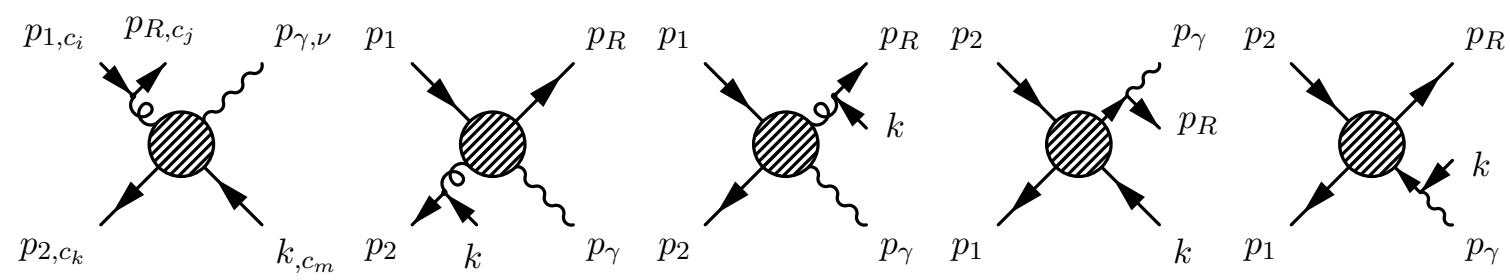

Figure 8. NLO Feynman diagrams for the NLP contributions of the process $q\left(p_{1}\right) \bar{q}\left(p_{2}\right) \rightarrow$ $q\left(p_{R}\right) \bar{q}(k) \gamma\left(p_{\gamma}\right)$.

Here we have separated the various contributions, which exhibit terms at different logarithmic orders and powers in the threshold expansion. In particular, it is interesting to note the NLL LP contribution $\Sigma_{F F, F F}$, which has arisen from the soft quark emission operator acting in the final state. This term is at LP due to the collinear nature of the quarkantiquark pair, but it is beyond the LL accuracy that is under control in our formalism. Furthermore, there are two types of contributions $\left(\Sigma_{I, I}\right.$ and $\left.\Sigma_{F, F}\right)$ whose NLP contribution can easily be guessed from the collinear pole. Interestingly, we also observe an interference term that contributes at LL NLP level: $\Sigma_{I, F}$. This term can be regarded as arising from the wide-angle emission of a soft quark. The contributions where a soft quark emission from an observed final state parton interferes with a similar emission from an unobserved parton vanish up to $\mathcal{O}(1)$ and will therefore only contribute beyond LL NLP.

Putting everything together, the NLP differential cross-section for this subprocess is

$$
\begin{aligned}
& v w(1-v) s \frac{\mathrm{d} \sigma_{q \bar{q} \rightarrow q \bar{q} \gamma}^{\mathrm{NLP}}}{\mathrm{d} v \mathrm{~d} w}= \\
& Q_{q}^{2} \alpha \alpha_{s}^{2}\left[\frac{1}{\varepsilon}\left\{-\frac{C_{F}}{C_{A}^{2}}(v(3(v-1) v+1))-\frac{C_{F}}{C_{A}} \frac{T_{q \bar{q}}(2(v-1) v((v-1) v+1)+3)}{2(1-v)}\right\}\right. \\
& +\ln (1-w)\left\{\frac{C_{F}}{C_{A}^{2}} v(1-2 v)^{2}+\frac{C_{F}}{C_{A}} \frac{2(v-1) v((v-1) v(2(v-1) v+5)+7)+3}{2(1-v)}\right\} \\
& \left.+\frac{1}{(1-w)_{+}} \frac{C_{F}}{3 C_{A}} T_{q \bar{q}}+\mathcal{O}(1)\right] \text {. }
\end{aligned}
$$

This result is promising, in that it demonstrates that the quark emission operator that we have introduced in section 2.2 can be used to correctly obtain the LL NLP contribution to the NLO cross-section. Since the emission of a quark is already at NLP due to the momentum information that is carried by the spinor, we do not need the momentum shift of the LO matrix elements.

\section{$5.3 \quad q g \rightarrow q g \gamma$}

This is the only subprocess for NLO prompt photon production that contains NLP contributions due to both quark and gluon emission. Let us first consider the radiation of a gluon, where the diagrams that we need are shown in figure 9. The derivation of the (next-to-)soft gluon contribution is directly analogous to the $q \bar{q} \rightarrow g g \gamma$ (next-to-)soft gluon amplitude analysed previously, and hence will not be presented in full detail here. The 

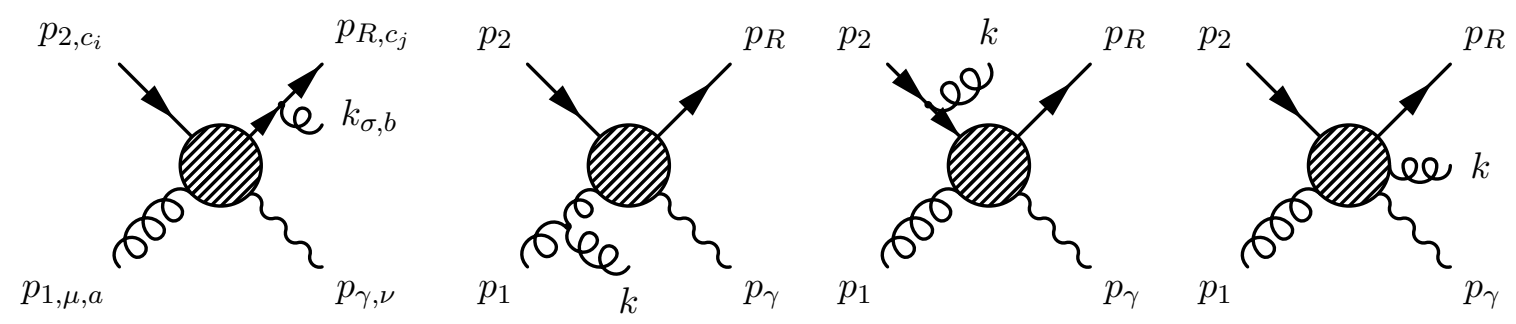

Figure 9. NLO Feynman diagrams for the NLP contributions of the process $g\left(p_{1}\right) q\left(p_{2}\right) \rightarrow$ $q\left(p_{R}\right) g(k) \gamma\left(p_{\gamma}\right)$.

result is

$$
\begin{aligned}
\left\langle\left|\mathcal{A}_{\mathrm{LP}+\mathrm{NLP}, q g \rightarrow \gamma q g}\right|^{2}\right\rangle=\frac{Q_{q}^{2} g_{s}^{4} g_{\mathrm{EM}}^{2}}{8 C_{A}(1-\varepsilon)}\left[\left(C_{F}-\frac{1}{2} C_{A}\right) \frac{2 p_{1} \cdot p_{R}}{\left(p_{1} \cdot k\right)\left(p_{R} \cdot k\right)}\right. & \times H_{g q \rightarrow q \gamma}\left(p_{1}+\delta p_{1 ; R}, p_{2}, p_{R}-\delta p_{R ; 1}, p_{\gamma}\right) \\
+ & \frac{1}{2} C_{A} \frac{2 p_{1} \cdot p_{2}}{\left(p_{1} \cdot k\right)\left(p_{2} \cdot k\right)} H_{g q \rightarrow q \gamma}\left(p_{1}+\delta p_{1 ; 2}, p_{2}+\delta p_{2 ; 1}, p_{R}, p_{\gamma}\right) \\
+ & \left.\frac{1}{2} C_{A} \frac{2 p_{2} \cdot p_{R}}{\left(p_{2} \cdot k\right)\left(p_{R} \cdot k\right)} H_{g q \rightarrow q \gamma}\left(p_{1}, p_{2}+\delta p_{2 ; R}, p_{R}-\delta p_{R ; 2}, p_{\gamma}\right)\right],
\end{aligned}
$$

where the factor of $1-\varepsilon$ in the common denominator stems from the fact that gluons can take $2(1-\varepsilon)$ different spin orientations in $d=4-2 \varepsilon$ dimensions. The expression for the LP+NLP soft gluon amplitude leads to the following differential cross-section, valid up to NLP:

$$
\begin{aligned}
v w(1-v) s \frac{\mathrm{d} \sigma_{q g \rightarrow \gamma q g}^{\mathrm{g}, \mathrm{LP}+\mathrm{NLP}}}{\mathrm{d} v \mathrm{~d} w}= & \frac{Q_{q}^{2} \alpha \alpha_{s}^{2}}{C_{A}}\left[-\frac{1}{\varepsilon} \frac{T_{q g}}{(1-w)_{+}}\left(C_{A}+C_{F}\right)\right. \\
& +\frac{1}{\varepsilon}\left\{C_{F} T_{q g}-C_{A} \frac{v(v(v(2 v-5)+4)-2)}{1-v}\right\} \\
& +\left(\frac{\ln (1-w)}{1-w}\right)_{+}\left(2 C_{A}+C_{F}\right) T_{q g} \\
& +\frac{1}{(1-w)_{+}}\left\{T_{q g}\left(C_{F} \ln \left(v^{2}(1-v)\right)+2 C_{A} \ln v-2\left(C_{F}+C_{A}\right) \ln \frac{\bar{\mu}^{2}}{s}\right)\right. \\
& \left.+C_{A} v^{3}+2 C_{F} v(v-1)\right\} \\
& -\ln (1-w)\left\{C_{F} T_{q g}+C_{A} \frac{v(v((18-7 v) v-16)+8)}{2(1-v)}\right\} \\
& +\mathcal{O}(\delta(1-w))+\mathcal{O}(1)],
\end{aligned}
$$

where $T_{q g}=v\left(1+(1-v)^{2}\right)$.

Next, we need the soft quark radiative contribution, and there are three $2 \rightarrow 2$ hard scattering diagrams on which we can use the quark emission operator $\mathcal{Q}_{j}$ to turn it into the process $q g \rightarrow q g \gamma$. These processes are $q g \rightarrow q \gamma, q \bar{q} \rightarrow g \gamma$ and $q g \rightarrow q g$. As in 


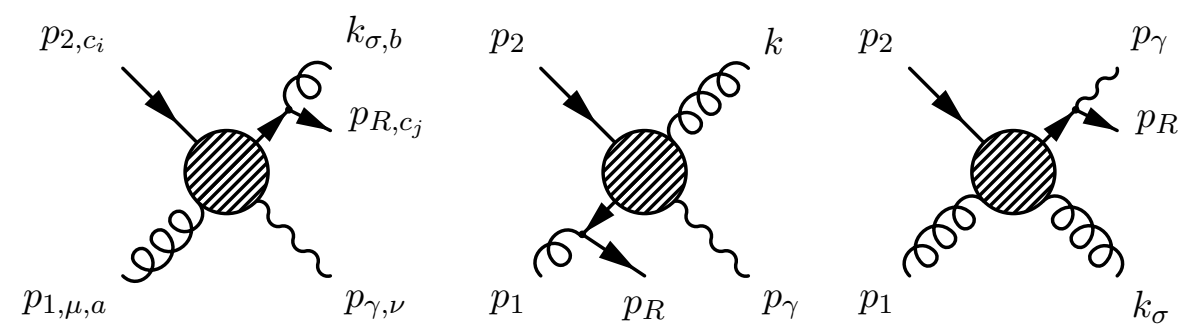

Figure 10. Feynman diagrams for the NLP contributions of the process $g\left(p_{1}\right) q\left(p_{2}\right) \rightarrow$ $q\left(p_{R}\right) g(k) \gamma\left(p_{\gamma}\right)$.

section 5.2, we will only select the resulting Feynman diagrams that describe the $q g \rightarrow q g \gamma$ sub-process. The generated NLP Feynman diagrams are given in figure 10, and the soft quark amplitude then consists of three pieces:

$$
\begin{aligned}
\mathcal{A}_{\mathrm{NLP}, \mathrm{quarks}}= & -\frac{i g_{s} t_{c_{j} c_{k}}^{b}}{2 k \cdot p_{R}} \epsilon_{\sigma}^{*}(k) \bar{u}\left(p_{R}\right) \gamma^{\sigma} \not k \mathcal{M}_{c_{k} c_{i}, g q \rightarrow \gamma(q)}^{a}\left(p_{1}, p_{2}, p_{\gamma}, k\right) \\
& -\frac{i g_{s} t_{c_{j} c_{k}}^{a}}{2 p_{1} \cdot p_{R}} \epsilon_{\mu}\left(p_{1}\right) \bar{u}\left(p_{R}\right) \gamma^{\mu} p_{1} \mathcal{M}_{c_{k} c_{i},(\bar{q}) q \rightarrow \gamma g}^{b}\left(p_{1}, p_{2}, p_{\gamma}, k\right) \\
& -\frac{i g_{\mathrm{EM}} Q_{q}}{2 p_{\gamma} \cdot p_{R}} \epsilon_{\nu}^{*}\left(p_{\gamma}\right) \bar{u}\left(p_{R}\right) \gamma^{\nu} \not p_{\gamma} \mathcal{M}_{c_{j} c_{i}, g q \rightarrow(q) g}^{a b}\left(p_{1}, p_{2}, p_{\gamma}, k\right) \\
\equiv & \mathcal{A}_{F F}+\mathcal{A}_{I}+\mathcal{A}_{F} .
\end{aligned}
$$

In section 5.1, we discussed the need to potentially include external ghost contributions when summing over all gluon polarisations in the final state. In that previous case, these contributions were absent at NLP. Here they will contribute owing to the presence of two hard gluons, as the quark is already soft and in order to observe the photon it needs to recoil against at least one other hard particle in the final state. The complete quark NLP cross-section then turns out to be:

$$
v w(1-v) s \frac{\mathrm{d} \sigma_{q g \rightarrow q g \gamma}^{\mathrm{q}, \mathrm{NLP}}}{\mathrm{d} v \mathrm{~d} w} \equiv \frac{Q_{q}^{2} \alpha \alpha_{s}^{2}}{C_{A}}\left[\Sigma_{I, I}+\Sigma_{I, F}+\Sigma_{I, F F}+\Sigma_{F, F}+\Sigma_{F, F F}+\Sigma_{F F, F F}\right],
$$

where the various contributions are as follows:

$$
\begin{aligned}
\Sigma_{I, I}= & -\frac{1}{\varepsilon} \frac{C_{F}}{C_{A}} \frac{v(2 v(v-1)+1)}{2(1-v)}+\ln (1-w) \frac{C_{F}}{C_{A}} \frac{v(2 v(v-1)+1)}{2(1-v)}+\mathcal{O}(1) \\
\Sigma_{I, F}= & \ln (1-w)\left\{\frac{C_{F}}{C_{A}} v^{3}+\frac{v^{4}}{2(1-v)}\right\}+\mathcal{O}(1) \\
\Sigma_{I, F F}= & \mathcal{O}(1) \\
\Sigma_{F, F}= & -\frac{1}{\varepsilon}\left\{\frac{1}{2} \frac{C_{F}}{C_{A}} v(1-v)\left(v^{2}+1\right)+\frac{1}{2} \frac{v^{2}\left(v^{2}+1\right)}{(1-v)}\right\} \\
& +\ln (1-w)\left\{\frac{1}{2} \frac{C_{F}}{C_{A}} v(1-v)\left(v^{2}+1\right)+\frac{1}{2} \frac{v^{2}\left(v^{2}+1\right)}{(1-v)}\right\}+\mathcal{O}(1) \\
\Sigma_{F, F F}= & \mathcal{O}(1) \\
\Sigma_{F F, F F}= & \frac{C_{F}}{4 C_{A}} \frac{T_{q g}}{(1-w)_{+}}+\mathcal{O}(1) .
\end{aligned}
$$


The complete NLP differential cross-section for the $q g \rightarrow q g \gamma$ subprocess, where one additional quark is radiated, is

$$
\begin{aligned}
v w(1-v) s \frac{\mathrm{d} \sigma_{q g \rightarrow q g \gamma}^{\mathrm{q}, \mathrm{NLP}}}{\mathrm{d} v \mathrm{~d} w}= & \frac{Q_{q}^{2} \alpha \alpha_{s}^{2}}{C_{A}}\left[-\frac{1}{\varepsilon}\left\{C_{F} \frac{v(v(v((v-2) v+4)-4)+2)}{2(1-v)}+C_{A} \frac{v^{2}\left(v^{2}+1\right)}{2(1-v)}\right\}\right. \\
& +\ln (1-w)\left\{C_{F} \frac{v((v-2) v((v-2) v+2)+2)}{2(1-v)}+C_{A} \frac{v\left(2 v^{3}+v\right)}{2(1-v)}\right\} \\
& \left.+\frac{1}{(1-w)_{+}} \frac{C_{F}}{4} T_{q g}+\mathcal{O}(1)\right] .
\end{aligned}
$$

We may now combine the gluon and quark radiative contributions, where the sum of eqs. (5.31) and (5.35) yields

$$
\begin{aligned}
v w(1-v) s \frac{\mathrm{d} \sigma_{q g \rightarrow q g \gamma}^{\mathrm{LP}+\mathrm{NLP}}}{\mathrm{d} v \mathrm{~d} w}= & \frac{Q_{q}^{2} \alpha \alpha_{s}^{2}}{C_{A}}\left[-\frac{1}{\varepsilon} \frac{1}{(1-w)_{+}}\left(C_{F}+C_{A}\right) T_{q g}\right. \\
& +\frac{1}{\varepsilon}\left\{C_{A} \frac{v}{2}(5(v-1) v+4)-C_{F} \frac{v\left(v\left(v^{3}-2 v+4\right)-2\right)}{2(1-v)}\right\} \\
& +\left(\frac{\ln (1-w)}{1-w}\right)_{+}\left(2 C_{A}+C_{F}\right) T_{q g} \\
& +\frac{1}{(1-w)_{+}}\left\{T_{q g}\left(C_{F} \ln \left((1-v) v^{2}\right)+2 C_{A} \ln v-2\left(C_{F}+C_{A}\right) \ln \frac{\bar{\mu}^{2}}{s}\right)\right. \\
& \left.+C_{A} v^{3}+\frac{C_{F}}{4} v\left(v^{2}+6 v-6\right)\right\} \\
& \left.+\ln (1-w)\left\{-C_{A} \frac{v}{2}(9(v-1) v+8)+C_{F} \frac{v\left(v\left((v-2) v^{2}+4\right)-2\right)}{2(1-v)}\right\}\right]
\end{aligned}
$$

This agrees to LL order with the full NLO calculation truncated at NLP, as presented here in appendix C. Our result shows that we can separately treat the (next-to-)soft gluon and soft fermion radiation, as is implied by our general analysis in section 2 .

We have presented here three of a total of seven different partonic subchannels for prompt photon production at NLO. The remaining channels work analogously to the ones already presented, and are listed in appendix B. We also present results for the unsubtracted NLO cross-sections (up to NLP) in appendix C, given that these have not previously been presented in the literature.

\section{Discussion}

In this paper, we have examined the origin of leading-logarithmic (LL) next-to-leadingpower (NLP) threshold terms at next-to-leading order (NLO) in perturbation theory. Our starting point was a previously obtained universal formula for the NLO cross-section for the production of an arbitrary number of colour-singlet particles [60], and we have generalised this in two ways. Firstly, we have considered procceses in which final state (massless) 
coloured particles at LO may be present. Secondly, we have considered contributions from other partonic channels, specifically those in which additional (anti)-quarks may be emitted.

The approach of ref. [60] is based on the soft expansion, in which one systematically expands the amplitude in the 4-momentum of the emitted radiation. This no longer coincides with the threshold expansion when final state jets are present. However, the dominant NLP contributions (i.e. those at leading logarithmic (LL) order) are correctly captured by the next-to-soft formalism for processes were only gluons emissions are present. ${ }^{11}$ We have explicitly verified this, and provided formulae in which the LL NLP terms in a general NLO cross-section are given in terms of the LO amplitude, with a simple momentum shift, as found for colour singlet production in ref. [60].

We illustrated our results using DIS, hadroproduction in electron-positron annihilation and prompt photon production. Due to the presence of unobserved quarks in the final state for all considered processes, we need to complement the next-to-soft gluon amplitude with soft quark amplitudes, which also give rise to LL NLP contributions. These can be treated completely independently from the next-to-soft gluon amplitude, and themselves factorise in terms of universal quark emission operators, that we have defined. Adding these soft quark contributions to the next-to-soft gluon amplitude shows that we are able to capture all LL NLP behaviour. For the prompt-photon process, we furthermore see a soft quark interference contribution, due to the presence of more than two coloured partons in the hard scattering. We have also clarified that leading logarithmic terms at NLP in a conjugate space, like Mellin space, may arise from the mass factorisation procedure, which would need to be kept track of in potential numerical studies.

We expect that our results will be of practical use, both in estimating higher-order cross-sections at NLO where these are unavailable, and in improving the stability of numerical codes. Work on examining these issues - including detailed numerical studies of the importance of NLP effects in specific collider processes of interest - is ongoing.

Note added. In the final stages of preparing this paper, ref. [75] appeared, which addresses the emission of soft and collinear radiation (including quarks) up to next-to-leading power, within the framework of soft collinear effective theory.

\section{Acknowledgments}

This article is based upon work from COST Action CA16201 PARTICLEFACE supported by COST (European Cooperation in Science and Technology). CDW was supported by the Science and Technology Facilities Council (STFC) Consolidated Grant ST/P000754/1 "String theory, gauge theory \& duality", and by the European Union's Horizon 2020 research and innovation programme under the Marie Skłodowska-Curie grant agreement No. 764850 "SAGEX". WB, EL and MvB acknowledge support from the Dutch NWOI program 156, "Higgs as Probe and Portal". MvB also acknowledges support from the Christine Mohrmann Stipendium. We wish to thank Lorenzo Magnea, Jort Sinninghe

\footnotetext{
${ }^{11}$ See refs. $[19,21,57,58,74]$ for similar conclusions in a SCET context.
} 
Damsté, Leonardo Vernazza and the participants of the 2018 Nikhef workshop "Next-toleading power corrections in particle physics" for useful discussions.

\section{A Useful definitions}

In this appendix, we collect useful formulae relating to the action of various operators appearing in eqs. (2.13), (2.18), as well as figure 3. First, in eq. (2.13), repeated here for convenience

$$
\mathcal{A}_{\mathrm{NLP}}=\sum_{j=1}^{n+2} \frac{g_{s} \mathbf{T}_{j}}{2 p_{j} \cdot k}\left(\mathcal{O}_{\mathrm{scal}, j}^{\sigma}+\mathcal{O}_{\mathrm{spin}, j}^{\sigma}+\mathcal{O}_{\mathrm{orb}, j}^{\sigma}\right) \otimes i \mathcal{M}_{\mathrm{H}}\left(p_{1}, \ldots, p_{i}, \ldots, p_{n+2}\right) \epsilon_{\sigma}^{*}(k),
$$

we must consider a general colour generator $\mathbf{T}_{j}$ acting on an external parton line $j$. This leads to a colour factor dressing the nonradiative amplitude

$$
\mathbf{T}_{j} \equiv\left\{\begin{aligned}
t_{c_{j} c_{i}}^{c} & \text { for an incoming quark or outgoing antiquark with colour label } c_{i} ; \\
-t_{c_{i} c_{j}}^{c} & \text { for an outgoing quark or incoming antiquark with colour label } c_{i} ; \\
i f^{c a b} & \text { for an external gluon with colour label } a,
\end{aligned}\right.
$$

where $\left\{t_{i j}^{a}\right\}$ are components of a generator in the fundamental representation. Next, we collect results for the numerator of the scalar contribution appearing in eq. (2.13). This can be written as

$$
\mathcal{O}_{\mathrm{scal}, j}^{\sigma} \equiv\left(2 p_{j}^{\sigma}+\eta k^{\sigma}\right),
$$

where $\eta=+1(-1)$ for a hard emitting particle in the final (initial) state respectively. The spin contribution in eq. (2.13) can be written in the generic form

$$
\mathcal{O}_{\text {spin }, j}^{\sigma} \equiv 2 i k_{\alpha} \Sigma_{j}^{\sigma \alpha}
$$

Here, $\Sigma_{j}^{\sigma \alpha}$ is a Lorentz generator in the appropriate representation of parton $j$, and given in specific cases by

$$
\Sigma_{j}^{\sigma \alpha} \equiv\left\{\begin{array}{cl}
S^{\alpha \sigma} & \text { for an incoming or outgoing quark; } \\
S^{\sigma \alpha} & \text { for an incoming or outgoing antiquark; } \\
M^{\sigma \alpha, \mu \rho} & \text { for an incoming gluon carrying Lorentz index } \mu \\
M^{\sigma \alpha, \rho \mu} & \text { for an outgoing gluon carrying Lorentz index } \mu,
\end{array}\right.
$$

where the relevant generators are defined in eqs. (2.2) and (2.5). The third term in eq. (2.13) can be written as

$$
\mathcal{O}_{\text {orb }, j}^{\sigma} \equiv 2 k_{\alpha} i L_{j}^{\sigma \alpha},
$$

where $L_{j}^{\sigma \alpha}$ is the orbital angular momentum operator of parton $j$, defined by

$$
L_{j}^{\sigma \alpha} \equiv \begin{cases}i\left(p_{j}^{\sigma} \frac{\partial}{\partial p_{j \alpha}}-p_{j}^{\alpha} \frac{\partial}{\partial p_{j \sigma}}\right) & \text { for an initial state emission } \\ i\left(p_{j}^{\alpha} \frac{\partial}{\partial p_{j \sigma}}-p_{j}^{\sigma} \frac{\partial}{\partial p_{j \alpha}}\right) & \text { for a final state emission. }\end{cases}
$$


Considering now the emission of soft quarks, the next-to-soft amplitude of eq. (2.18), repeated for convenience here

$$
\mathcal{A}_{\mathrm{NLP}, \mathcal{Q}}=\sum_{j=1}^{n+2} \frac{g_{s}}{2 p_{j} \cdot k} \mathcal{Q}_{j} \otimes i \mathcal{M}_{j}\left(p_{1}, p_{2}, \ldots, p_{j}, \ldots, p_{n+2}\right),
$$

contains the quark emission operator $\mathcal{Q}_{j}$, whose action on all possible species of incoming/outgoing parton legs is depicted in figure 3. In terms of the amplitude, we may think of this operator as acting on the wavefunction for leg $j$, as follows:

$$
\begin{aligned}
\mathcal{Q}_{j}\left(u\left(p_{j}\right)\right) & =t_{c_{j} c_{m}}^{a} \epsilon_{\mu}\left(p_{j}\right) \not p_{j} \gamma^{\mu} v(k) \\
\mathcal{Q}_{j}\left(\bar{u}\left(p_{j}\right)\right) & =-t_{c_{m} c_{j}}^{a} \epsilon_{\mu}^{*}\left(p_{j}\right) \bar{u}(k) \gamma^{\mu} \not p_{j} \\
\mathcal{Q}_{j}\left(v\left(p_{j}\right)\right) & =t_{c_{j} c_{m}}^{a} \epsilon_{\mu}^{*}\left(p_{j}\right) \not p_{j} \gamma^{\mu} v(k) \\
\mathcal{Q}_{j}\left(\bar{v}\left(p_{j}\right)\right) & =-t_{c_{m} c_{j}}^{a} \epsilon_{\mu}\left(p_{j}\right) \bar{u}(k) \gamma^{\mu} \not p_{j} \\
\mathcal{Q}_{j}\left(\epsilon_{\mu}\left(p_{j}\right)\right) & =-\left(t_{c_{m} c_{j}}^{a} \bar{u}(k) \gamma_{\mu} u\left(p_{j}\right)+t_{c_{j} c_{m}}^{a} \bar{v}\left(p_{j}\right) \gamma_{\mu} v(k)\right) \\
\mathcal{Q}_{j}\left(\epsilon_{\mu}^{*}\left(p_{j}\right)\right) & =t_{c_{j} c_{m}}^{a} \bar{u}\left(p_{j}\right) \gamma_{\mu} v(k)+t_{c_{m} c_{j}}^{a} \bar{u}(k) \gamma_{\mu} v\left(p_{j}\right) .
\end{aligned}
$$

There are a couple of further subtleties regarding how to apply this operator in practice. Firstly, in cases where $p_{j}$ is an initial state particle, or is explicitly observed in the final state (i.e. in an observable that is defined in a way that is not fully inclusive), one must only include those contributions arising from the $\mathcal{Q}_{j}$ operator such that the (observed) parton appearing in the LO process has the hard momentum. Secondly, in the final line of figure 3, one includes the possibility that either the quark or antiquark is soft. If neither of the decay products of the gluon are explicitly observed, but instead summed over inclusively, then one double counts the quark/antiquark emission contribution due to the integration over all possible momenta $k$. This double counting must then be corrected for by a factor of $1 / 2$. Thirdly, the polarisation vector may also belong to a photon. In this case, the coupling that appears in eq. (2.18) should be modified to $g_{\mathrm{EM}}$ and the generator becomes $Q_{q}$. We see an explicit example of these subtleties in the prompt photon analysis of section 5 .

\section{B Results for the remaining channels}

Here we report the results of the prompt photon channels that are not discussed in section 5 . All of the remaining channels only have quarks in the final state, hence only the soft quark formalism is needed to derive these results. The general form of the obtained NLP crosssection is

$$
v w(1-v) s \frac{\mathrm{d} \sigma_{\mathrm{q}}^{\mathrm{NLP}}}{\mathrm{d} v \mathrm{~d} w} \equiv \alpha \alpha_{s}^{2}\left[\Sigma_{I, I}+\Sigma_{I, F}+\Sigma_{I, F F}+\Sigma_{F, F}+\Sigma_{F, F F}+\Sigma_{F F, F F}\right],
$$

where $I$ indicates that the contribution stems from initial state radiation, and $F(F F)$ indicates that the contribution stems from final state radiation where the other particle is (un)observed. 
The separate contributions for the $g g \rightarrow q \bar{q} \gamma$ sub-process are

$$
\begin{aligned}
\Sigma_{I, I} & =\frac{v Q_{q}^{2}}{2 C_{A} C_{F}}\left[-\frac{1}{\varepsilon} C_{F}(2(v-1) v+3)-\ln (1-w)\left(C_{A}-C_{F}(2(v-1) v+5)\right)+\mathcal{O}(1)\right] \\
\Sigma_{F, F} & =\frac{v\left(C_{A}(v-1) v+C_{F}\right) Q_{q}^{2}}{2 C_{A} C_{F}}\left[-\frac{1}{\varepsilon}(2(v-1) v+1)+\log (1-w)(2(v-1) v+1)+\mathcal{O}(1)\right] \\
\Sigma_{I, F} & =\frac{Q_{q}^{2} v}{2 C_{A} C_{F}}\left[\log (1-w)\left(-C_{A}(3(v-1) v+1)+2 C_{F}(2(v-1) v+1)\right)+\mathcal{O}(1)\right] \\
\Sigma_{I, F F} & =\Sigma_{F F, F F}=\Sigma_{F, F F}=0,
\end{aligned}
$$

and those from the $q q \rightarrow q q \gamma$ sub-process read

$$
\begin{aligned}
& \Sigma_{I, I}=\frac{Q_{q}^{2} C_{F}}{2 C_{A}^{2}}[-\frac{1}{\varepsilon}\left\{\frac{C_{A}(2(v-1) v((v-1) v+1)+1)}{1-v}\right\} \\
&\left.+\ln (1-w)\left\{\frac{C_{A}(2(v-1) v((v-1) v+1)+1)}{1-v}+2 v\right\}+\mathcal{O}(1)\right] \\
& \Sigma_{F, F}=\frac{Q_{q}^{2} C_{F}}{C_{A}^{2}}\left[-\frac{1}{\varepsilon}\left\{\frac{C_{A}((v-1) v((v-1) v+3)+1)}{1-v}-v\right\}\right.\left.+\ln (1-w)\left\{\frac{C_{A}((v-1) v((v-1) v+3)+1)}{1-v}-v\right\}+\mathcal{O}(1)\right] \\
& \Sigma_{I, F}=\frac{Q_{q}^{2} C_{F}}{C_{A}^{2}}\left[\ln (1-w)\left\{v\left((2(v-1) v+3) C_{A}+2\right)-\frac{1}{1-v}\right\}+\mathcal{O}(1)\right] \\
& \Sigma_{I, F F}=\Sigma_{F F, F F}=\Sigma_{F, F F}=0 .
\end{aligned}
$$

For the $q q^{\prime} \rightarrow q q^{\prime} \gamma$ sub-process we find

$$
\begin{aligned}
\Sigma_{I, I}=\frac{C_{F}}{2 C_{A}}[ & \frac{1}{\varepsilon}\left\{Q_{q}^{2}\left(v^{2}+1\right)(v-1)-Q_{q^{\prime}}^{2} \frac{v^{2}((v-2) v+2)}{1-v}\right\} \\
& \left.-\ln (1-w)\left\{Q_{q}^{2}\left(v^{2}+1\right)(v-1)-Q_{q^{\prime}}^{2} \frac{v^{2}((v-2) v+2)}{1-v}\right\}+\mathcal{O}(1)\right] \\
\Sigma_{F, F}=\frac{C_{F}}{2 C_{A}} & {\left[\frac{1}{\varepsilon}\left\{Q_{q}^{2}((v-2) v+2)(v-1)-Q_{q^{\prime}}^{2} \frac{v^{2}\left(v^{2}+1\right)}{1-v}\right\}\right.} \\
& \left.-\ln (1-w)\left\{Q_{q}^{2}((v-2) v+2)(v-1)-Q_{q^{\prime}}^{2} \frac{v^{2}\left(v^{2}+1\right)}{1-v}\right\}+\mathcal{O}(1)\right] \\
\Sigma_{I, F}=\frac{Q_{q} Q_{q^{\prime}} C_{F} v}{C_{A}}[\ln (1-w)(2(v-1) v+3)+\mathcal{O}(1)] & \Sigma_{I, F F}=\Sigma_{F F, F F}=\Sigma_{F, F F}=0 .
\end{aligned}
$$


Finally, the separate contributions for the $q \bar{q} \rightarrow q^{\prime} \bar{q}^{\prime} \gamma$ sub-process are

$$
\begin{aligned}
& \Sigma_{F, F}=\frac{Q_{q^{\prime}}^{2} C_{F}}{C_{A}}[-\frac{1}{\varepsilon}(1-v) v^{2}\left(2 v^{2}-2 v+1\right) \\
&\left.+\ln (1-w)(1-v) v^{2}\left(2 v^{2}-2 v+1\right)+\mathcal{O}(1)\right] \\
& \Sigma_{F F, F F}=\frac{Q_{q}^{2} C_{F}}{C_{A}}\left[\frac{1}{(1-w)_{+}} \frac{T_{q \bar{q}}}{3}+\mathcal{O}(1)\right] \\
& \Sigma_{F, F F}=\mathcal{O}(1) \\
& \Sigma_{I, F}=\Sigma_{I, I}=\Sigma_{I, F F}=0 .
\end{aligned}
$$

These results are in full agreement with the exact NLO results at LL order, which are presented in appendix $\mathrm{C}$.

\section{NLO cross-section for prompt photon production}

In this appendix we write down the NLO cross-sections for the $q \bar{q} \rightarrow g g \gamma, q \bar{q} \rightarrow q \bar{q} \gamma$ and $q g \rightarrow q g \gamma$ processes, expanded up to NLP and before mass factorisation. The expressions for the cross-sections after mass factorisation can be found in ref. [72]. We will cast the cross-section for all sub-processes in the form

$$
\begin{aligned}
v w(1-v) s \frac{\mathrm{d} \sigma^{\mathrm{NLP}}}{\mathrm{d} v \mathrm{~d} w}=\alpha \alpha_{s}^{2}\left[c_{1}\right. & \frac{1}{\varepsilon} \frac{1}{(1-w)_{+}}+c_{2} \frac{1}{\varepsilon}+c_{3} \frac{1}{(1-w)_{+}}+c_{3}^{\prime} \frac{1}{(1-w)_{+}} \ln \frac{\bar{\mu}^{2}}{s} \quad(\mathrm{C} .1) \\
& \left.+c_{4}\left(\frac{\ln (1-w)}{1-w}\right)_{+}+c_{5} \ln (1-w)+\mathcal{O}(\delta(1-w))+\mathcal{O}(1)\right] .
\end{aligned}
$$

The coefficients for the $q \bar{q} \rightarrow g g \gamma$ sub-process (section 5.1) read

$$
\begin{aligned}
c_{1}= & -4 \frac{Q_{q}^{2} C_{F}^{2}}{C_{A}} T_{q \bar{q}} \\
c_{2}= & -2 \frac{Q_{q}^{2} C_{F}^{2}}{C_{A}} \frac{4(v-1)^{2} v-1}{1-v} \\
c_{3}= & \frac{Q_{q}^{2} C_{F}}{C_{A}}\left(8 C_{F}\left(((v-1) v+1)+T_{q \bar{q}} \ln v\right)+C_{A}\left(T_{q \bar{q}}\left(-\frac{11}{6}+2 \ln (1-v)\right)\right)\right) \\
c_{3}^{\prime}= & -8 \frac{Q_{q}^{2} C_{F}^{2}}{C_{A}} T_{q \bar{q}} \\
c_{4}= & 2 \frac{Q_{q}^{2} C_{F}}{C_{A}} T_{q \bar{q}}\left(4 C_{F}-C_{A}\right) \\
c_{5}= & \frac{Q_{q}^{2} C_{F}}{C_{A}}\left(4 C_{F}-C_{A}\right) \frac{4(v-1)^{2} v-1}{1-v},
\end{aligned}
$$


and those for the $q \bar{q} \rightarrow q \bar{q} \gamma$ sub-process (section 5.2):

$$
\begin{aligned}
& c_{1}=c_{3}^{\prime}=c_{4}=0 \\
& c_{2}=-\frac{Q_{q}^{2} C_{F}}{C_{A}^{2}}(v(3(v-1) v+1))-\frac{Q_{q}^{2} C_{F}}{C_{A}} \frac{T_{q \bar{q}}(2(v-1) v((v-1) v+1)+3)}{2(1-v)} \\
& c_{3}=\frac{Q_{q}^{2} C_{F}}{C_{A}} \frac{T_{q \bar{q}}}{3} \\
& c_{5}=\frac{Q_{q}^{2} C_{F}}{C_{A}^{2}} v(1-2 v)^{2}+\frac{Q_{q}^{2} C_{F}}{C_{A}} \frac{2(v-1) v((v-1) v(2(v-1) v+5)+7)+3}{2(1-v)} .
\end{aligned}
$$

Next, we have the coefficients for the $q g \rightarrow q g \gamma$ sub-process (section 5.3),

$$
\begin{aligned}
c_{1} & =-\frac{Q_{q}^{2}}{C_{A}} T_{q g}\left(C_{A}+C_{F}\right) \\
c_{2} & =\frac{v Q_{q}^{2}}{2 C_{A}}\left(C_{A}(5(v-1) v+4)-C_{F} \frac{v\left(v^{3}-2 v+4\right)-2}{1-v}\right) \\
c_{3} & =\frac{Q_{q}^{2}}{C_{A}}\left(C_{A}\left(v^{3}+2 T_{q g} \ln v\right)+C_{F}\left(T_{q g} \ln \left((1-v) v^{2}\right)+\frac{v}{4}\left(v^{2}+6 v-6\right)\right)\right) \\
c_{3}^{\prime} & =-\frac{2 Q_{q}^{2}}{C_{A}} T_{q g}\left(C_{A}+C_{F}\right) \\
c_{4} & =\frac{Q_{q}^{2}}{C_{A}}\left(2 C_{A}+C_{F}\right) T_{q g} \\
c_{5} & =\frac{v Q_{q}^{2}}{2 C_{A}(1-v)}\left(C_{A}(v-1)(9(v-1) v+8)+C_{F}\left(v\left((v-2) v^{2}+4\right)-2\right)\right)
\end{aligned}
$$

followed by the $g g \rightarrow q \bar{q} \gamma$ sub-process:

$$
\begin{aligned}
& c_{1}=c_{3}^{\prime}=c_{3}=c_{4}=0 \\
& c_{2}=\frac{v Q_{q}^{2}}{2 C_{A} C_{F}}\left(C_{A} v(v(-2 v(v-2)-3)+1)-4 C_{F}(v(v-1)+1)\right) \\
& c_{5}=\frac{v Q_{q}^{2}}{C_{A} C_{F}}\left(C_{A}\left(v^{4}-2 v^{3}+v-1\right)+4 C_{F}((v-1) v+1)\right) .
\end{aligned}
$$

For the $q q \rightarrow q q \gamma$ sub-process, we find

$$
\begin{aligned}
& c_{1}=c_{3}^{\prime}=c_{3}=c_{4}=0 \\
& c_{2}=\frac{Q_{q}^{2} C_{F}}{C_{A}^{2}}\left(v-C_{A} \frac{(2(v-1) v+1)(2(v-1) v+3)}{2(1-v)}\right) \\
& c_{5}=\frac{Q_{q}^{2} C_{F}}{C_{A}^{2}} \frac{C_{A}\left(2 v^{2}-2 v+3\right)-4 v^{2}+4 v-2}{2(1-v)},
\end{aligned}
$$

and for the $q q^{\prime} \rightarrow q q^{\prime} \gamma$ sub-process (note that ref. [72] has $Q_{q} \leftrightarrow Q_{q^{\prime}}$ as a result of an interchange in the assigned initial state momenta)

$$
\begin{aligned}
& c_{1}=c_{3}^{\prime}=c_{3}=c_{4}=0 \\
& c_{2}=-\frac{C_{F}}{C_{A}} \frac{2(v-1) v+3}{2(1-v)}\left(Q_{q}^{2}(1-v)^{2}+Q_{q^{\prime}}^{2} v^{2}\right) \\
& c_{5}=\frac{C_{F}}{C_{A}} \frac{2(v-1) v+3}{2(1-v)}\left(Q_{q}^{2}(1-v)^{2}+Q_{q} Q_{q^{\prime}} 2 v(1-v)+Q_{q^{\prime}}^{2} v^{2}\right) .
\end{aligned}
$$


Finally, the coefficients for the $q \bar{q} \rightarrow q^{\prime} \bar{q}^{\prime} \gamma$ sub-process are given by

$$
\begin{aligned}
& c_{1}=c_{3}^{\prime}=c_{4}=0 \\
& c_{3}=\frac{Q_{q}^{2} C_{F}}{C_{A}} \frac{T_{q \bar{q}}}{3} \\
& c_{2}=-\frac{C_{F}}{C_{A}} Q_{q^{\prime}}^{2}(1-v) v^{2}\left(2 v^{2}-2 v+1\right) \\
& c_{5}=\frac{C_{F}}{C_{A}} Q_{q^{\prime}}^{2}(1-v) v^{2}\left(2 v^{2}-2 v+1\right) .
\end{aligned}
$$

Open Access. This article is distributed under the terms of the Creative Commons Attribution License (CC-BY 4.0), which permits any use, distribution and reproduction in any medium, provided the original author(s) and source are credited.

\section{References}

[1] G. Parisi, Summing large perturbative corrections in QCD, Phys. Lett. 90B (1980) 295 [INSPIRE].

[2] G. Curci and M. Greco, Large infrared corrections in QCD processes, Phys. Lett. 92B (1980) 175 [INSPIRE].

[3] G.F. Sterman, Summation of large corrections to short distance hadronic cross-sections, Nucl. Phys. B 281 (1987) 310 [inSPIRE].

[4] S. Catani and L. Trentadue, Resummation of the QCD perturbative series for hard processes, Nucl. Phys. B 327 (1989) 323 [InSPIRE].

[5] S. Catani and L. Trentadue, Comment on QCD exponentiation at large $x$, Nucl. Phys. B 353 (1991) 183 [INSPIRE].

[6] J.G.M. Gatheral, Exponentiation of eikonal cross-sections in nonabelian gauge theories, Phys. Lett. 133B (1983) 90 [INSPIRE].

[7] J. Frenkel and J.C. Taylor, Nonabelian eikonal exponentiation, Nucl. Phys. B 246 (1984) 231 [INSPIRE].

[8] G. Sterman, Infrared divergences in perturbative QCD. AIP Conf. Proc. 74 (1981) 22.

[9] G.P. Korchemsky and G. Marchesini, Structure function for large $x$ and renormalization of Wilson loop, Nucl. Phys. B 406 (1993) 225 [hep-ph/9210281] [InSPIRE].

[10] G.P. Korchemsky and G. Marchesini, Resummation of large infrared corrections using Wilson loops, Phys. Lett. B 313 (1993) 433 [INSPIRE].

[11] S. Forte and G. Ridolfi, Renormalization group approach to soft gluon resummation, Nucl. Phys. B 650 (2003) 229 [hep-ph/0209154] [INSPIRE].

[12] H. Contopanagos, E. Laenen and G.F. Sterman, Sudakov factorization and resummation, Nucl. Phys. B 484 (1997) 303 [hep-ph/9604313] [inSPIRE].

[13] T. Becher and M. Neubert, Threshold resummation in momentum space from effective field theory, Phys. Rev. Lett. 97 (2006) 082001 [hep-ph/0605050] [INSPIRE].

[14] M.D. Schwartz, Resummation and NLO matching of event shapes with effective field theory, Phys. Rev. D 77 (2008) 014026 [arXiv:0709.2709] [INSPIRE]. 
[15] C.W. Bauer, S.P. Fleming, C. Lee and G.F. Sterman, Factorization of $e^{+} e^{-}$event shape distributions with hadronic final states in soft collinear effective theory, Phys. Rev. D 78 (2008) 034027 [arXiv:0801.4569] [INSPIRE].

[16] J.-y. Chiu, A. Fuhrer, R. Kelley and A.V. Manohar, Factorization structure of gauge theory amplitudes and application to hard scattering processes at the LHC, Phys. Rev. D 80 (2009) 094013 [arXiv: 0909.0012] [INSPIRE].

[17] M. Krämer, E. Laenen and M. Spira, Soft gluon radiation in Higgs boson production at the LHC, Nucl. Phys. B 511 (1998) 523 [hep-ph/9611272] [INSPIRE].

[18] F. Herzog and B. Mistlberger, The soft-virtual Higgs cross-section at $N^{3} L O$ and the convergence of the threshold expansion, in Proceedings, 49th Rencontres de Moriond on QCD and High Energy Interactions: La Thuile, Italy, March 22-29, 2014, pp. 57-60, 2014, arXiv: 1405.5685 [INSPIRE].

[19] I. Moult, L. Rothen, I.W. Stewart, F.J. Tackmann and H.X. Zhu, Subleading power corrections for N-jettiness subtractions, Phys. Rev. D 95 (2017) 074023 [arXiv:1612.00450] [INSPIRE].

[20] R. Boughezal, X. Liu and F. Petriello, Power corrections in the N-jettiness subtraction scheme, JHEP 03 (2017) 160 [arXiv:1612.02911] [INSPIRE].

[21] I. Moult, L. Rothen, I.W. Stewart, F.J. Tackmann and H.X. Zhu, $N$-jettiness subtractions for $g g \rightarrow H$ at subleading power, Phys. Rev. D 97 (2018) 014013 [arXiv:1710.03227] [INSPIRE].

[22] R. Boughezal, A. Isgrò and F. Petriello, Next-to-leading-logarithmic power corrections for $N$-jettiness subtraction in color-singlet production, Phys. Rev. D 97 (2018) 076006 [arXiv: 1802.00456] [INSPIRE].

[23] M.A. Ebert, I. Moult, I.W. Stewart, F.J. Tackmann, G. Vita and H.X. Zhu, Power Corrections for $N$-Jettiness Subtractions at $\mathcal{O}\left(\alpha_{s}\right)$, JHEP 12 (2018) 084 [arXiv:1807.10764] [INSPIRE].

[24] M.A. Ebert, I. Moult, I.W. Stewart, F.J. Tackmann, G. Vita and H.X. Zhu, Subleading power rapidity divergences and power corrections for $q_{T}$, JHEP 04 (2019) 123 [arXiv: 1812.08189] [INSPIRE].

[25] F.E. Low, Bremsstrahlung of very low-energy quanta in elementary particle collisions, Phys. Rev. 110 (1958) 974 [INSPIRE].

[26] T.H. Burnett and N.M. Kroll, Extension of the low soft photon theorem, Phys. Rev. Lett. 20 (1968) 86 [INSPIRE].

[27] V. Del Duca, High-energy bremsstrahlung theorems for soft photons, Nucl. Phys. B 345 (1990) 369 [INSPIRE].

[28] E. Laenen, G. Stavenga and C.D. White, Path integral approach to eikonal and next-to-eikonal exponentiation, JHEP 03 (2009) 054 [arXiv:0811.2067] [INSPIRE].

[29] E. Laenen, L. Magnea, G. Stavenga and C.D. White, Next-to-eikonal corrections to soft gluon radiation: a diagrammatic approach, JHEP 01 (2011) 141 [arXiv:1010.1860] [INSPIRE].

[30] G. Soar, S. Moch, J.A.M. Vermaseren and A. Vogt, On Higgs-exchange DIS, physical evolution kernels and fourth-order splitting functions at large $x$, Nucl. Phys. B $\mathbf{8 3 2}$ (2010) 152 [arXiv: 0912.0369] [INSPIRE]. 
[31] S. Moch and A. Vogt, On non-singlet physical evolution kernels and large-x coefficient functions in perturbative QCD, JHEP 11 (2009) 099 [arXiv:0909.2124] [INSPIRE].

[32] S. Moch and A. Vogt, Threshold resummation of the structure function $F_{L}$, JHEP 04 (2009) 081 [arXiv: 0902.2342] [INSPIRE].

[33] D. de Florian, J. Mazzitelli, S. Moch and A. Vogt, Approximate $N^{3}$ LO Higgs-boson production cross section using physical-kernel constraints, JHEP 10 (2014) 176 [arXiv: 1408.6277] [INSPIRE].

[34] N.A. Lo Presti, A.A. Almasy and A. Vogt, Leading large-x logarithms of the quark 85 gluon contributions to inclusive Higgs-boson and lepton-pair production, Phys. Lett. B 737 (2014) 120 [arXiv: 1407.1553] [inSPIRE].

[35] E. Laenen, L. Magnea and G. Stavenga, On next-to-eikonal corrections to threshold resummation for the Drell-Yan and DIS cross sections, Phys. Lett. B 669 (2008) 173 [arXiv: 0807.4412] [INSPIRE].

[36] G. Grunberg, Threshold resummation to any order in (1-x), arXiv:0710.5693 [INSPIRE].

[37] G. Grunberg and V. Ravindran, On threshold resummation beyond leading 1-x order, JHEP 10 (2009) 055 [arXiv: 0902 .2702] [inSPIRE].

[38] G. Grunberg, Large-x structure of physical evolution kernels in Deep Inelastic Scattering, Phys. Lett. B 687 (2010) 405 [arXiv:0911.4471] [InSPIRE].

[39] F. Cachazo and A. Strominger, Evidence for a new soft graviton theorem, arXiv:1404.4091 [INSPIRE].

[40] E. Casali, Soft sub-leading divergences in Yang-Mills amplitudes, JHEP 08 (2014) 077 [arXiv: 1404.5551] [INSPIRE].

[41] C.D. White, Factorization properties of soft graviton amplitudes, JHEP 05 (2011) 060 [arXiv: 1103.2981] [INSPIRE].

[42] H. Gervais, Soft photon theorem for high energy amplitudes in Yukawa and scalar theories, Phys. Rev. D 95 (2017) 125009 [arXiv:1704.00806] [INSPIRE].

[43] H. Gervais, Soft graviton emission at high and low energies in Yukawa and scalar theories, Phys. Rev. D 96 (2017) 065007 [arXiv:1706.03453] [INSPIRE].

[44] H. Gervais, Soft radiation theorems at all loop order in quantum field theory, Ph.D. thesis, SUNY, Stony Brook, U.S.A. (4 August 2017).

[45] D. Bonocore, E. Laenen, L. Magnea, S. Melville, L. Vernazza and C.D. White, A factorization approach to next-to-leading-power threshold logarithms, JHEP 06 (2015) 008 [arXiv: 1503.05156] [INSPIRE].

[46] D. Bonocore, E. Laenen, L. Magnea, L. Vernazza and C.D. White, Non-abelian factorisation for next-to-leading-power threshold logarithms, JHEP 12 (2016) 121 [arXiv:1610.06842] [INSPIRE].

[47] M. Beneke, F. Campanario, T. Mannel and B.D. Pecjak, Power corrections to $\bar{B} \rightarrow X_{u} l \bar{\nu}\left(X_{s} \gamma\right)$ decay spectra in the 'shape-function' region, JHEP 06 (2005) 071 [hep-ph/0411395] [INSPIRE].

[48] A.J. Larkoski, D. Neill and I.W. Stewart, Soft theorems from effective field theory, JHEP 06 (2015) 077 [arXiv: 1412.3108] [INSPIRE]. 
[49] D.W. Kolodrubetz, I. Moult and I.W. Stewart, Building blocks for subleading helicity operators, JHEP 05 (2016) 139 [arXiv: 1601.02607] [INSPIRE].

[50] I. Moult, I.W. Stewart and G. Vita, A subleading operator basis and matching for $g g \rightarrow H$, JHEP 07 (2017) 067 [arXiv: 1703.03408] [INSPIRE].

[51] I. Feige, D.W. Kolodrubetz, I. Moult and I.W. Stewart, A complete basis of helicity operators for subleading factorization, JHEP 11 (2017) 142 [arXiv:1703.03411] [INSPIRE].

[52] C.-H. Chang, I.W. Stewart and G. Vita, A subleading power operator basis for the scalar quark current, JHEP 04 (2018) 041 [arXiv: 1712.04343] [INSPIRE].

[53] M. Beneke, M. Garny, R. Szafron and J. Wang, Anomalous dimension of subleading-power $N$-jet operators, JHEP 03 (2018) 001 [arXiv:1712.04416] [inSPIRE].

[54] R. Goerke and M. Luke, Power Counting and Modes in SCET, JHEP 02 (2018) 147 [arXiv: 1711.09136] [INSPIRE].

[55] R. Goerke and M. Inglis-Whalen, Renormalization of dijet operators at order $1 / Q^{2}$ in soft-collinear effective theory, JHEP 05 (2018) 023 [arXiv:1711.09147] [INSPIRE].

[56] M. Beneke, M. Garny, R. Szafron and J. Wang, Anomalous dimension of subleading-power $N$-jet operators. Part II, JHEP 11 (2018) 112 [arXiv:1808.04742] [INSPIRE].

[57] M. Beneke, A. Broggio, M. Garny, S. Jaskiewicz, R. Szafron, L. Vernazza et al., Leading-logarithmic threshold resummation of the Drell-Yan process at next-to-leading power, JHEP 03 (2019) 043 [arXiv: 1809.10631] [INSPIRE].

[58] I. Moult, I.W. Stewart, G. Vita and H.X. Zhu, First Subleading Power Resummation for Event Shapes, JHEP 08 (2018) 013 [arXiv: 1804.04665] [INSPIRE].

[59] N. Bahjat-Abbas, D. Bonocore, J. Sinninghe Damsté, E. Laenen, L. Magnea, L. Vernazza et al., Diagrammatic resummation of leading-logarithmic threshold effects at next-to-leading power, JHEP 11 (2019) 002 [arXiv: 1905.13710] [INSPIRE].

[60] V. Del Duca, E. Laenen, L. Magnea, L. Vernazza and C.D. White, Universality of next-to-leading power threshold effects for colourless final states in hadronic collisions, JHEP 11 (2017) 057 [arXiv: 1706.04018] [INSPIRE].

[61] D. Bonocore, E. Laenen, L. Magnea, L. Vernazza and C.D. White, The method of regions and next-to-soft corrections in Drell-Yan production, Phys. Lett. B 742 (2015) 375 [arXiv: 1410.6406] [INSPIRE].

[62] N. Bahjat-Abbas, J. Sinninghe Damsté, L. Vernazza and C.D. White, On next-to-leading power threshold corrections in Drell-Yan production at $N^{3} L O$, JHEP 10 (2018) 144 [arXiv: 1807.09246] [INSPIRE].

[63] S. Alte, M. König and M. Neubert, Effective Field Theory after a New-Physics Discovery, JHEP 08 (2018) 095 [arXiv: 1806. 01278] [INSPIRE].

[64] M. Beneke and V.A. Smirnov, Asymptotic expansion of Feynman integrals near threshold, Nucl. Phys. B 522 (1998) 321 [hep-ph/9711391] [InSPIRE].

[65] C.D. White, Diagrammatic insights into next-to-soft corrections, Phys. Lett. B 737 (2014) 216 [arXiv: 1406.7184] [INSPIRE].

[66] Z. Bern, S. Davies, P. Di Vecchia and J. Nohle, Low-energy behavior of gluons and gravitons from gauge invariance, Phys. Rev. D 90 (2014) 084035 [arXiv:1406.6987] [INSPIRE]. 
[67] J. Broedel, M. de Leeuw, J. Plefka and M. Rosso, Constraining subleading soft gluon and graviton theorems, Phys. Rev. D 90 (2014) 065024 [arXiv:1406.6574] [InSPIRE].

[68] S. Weinberg, Infrared photons and gravitons, Phys. Rev. 140 (1965) B516 [InSPIRE].

[69] E.B. Zijlstra and W.L. van Neerven, Second order QCD corrections to deep inelastic scattering, Nucl. Phys. Proc. Suppl. B 29 (1992) 131.

[70] E.B. Zijlstra and W.L. van Neerven, Order $\alpha_{s}^{2} Q C D$ corrections to the deep inelastic proton structure functions $F_{2}$ and $F_{L}$, Nucl. Phys. B 383 (1992) 525 [InSPIRE].

[71] P.J. Rijken and W.L. van Neerven, Higher order QCD corrections to the transverse and longitudinal fragmentation functions in electron-positron annihilation, Nucl. Phys. B 487 (1997) 233 [hep-ph/9609377] [INSPIRE].

[72] L.E. Gordon and W. Vogelsang, Polarized and unpolarized prompt photon production beyond the leading order, Phys. Rev. D 48 (1993) 3136 [InSPIRE].

[73] S. Catani, M.L. Mangano and P. Nason, Sudakov resummation for prompt photon production in hadron collisions, JHEP 07 (1998) 024 [hep-ph/9806484] [INSPIRE].

[74] A. Bhattacharya, I. Moult, I.W. Stewart and G. Vita, Helicity Methods for High Multiplicity Subleading Soft and Collinear Limits, JHEP 05 (2019) 192 [arXiv: 1812.06950] [INSPIRE].

[75] I. Moult, I.W. Stewart and G. Vita, Subleading Power Factorization with Radiative Functions, JHEP 11 (2019) 153 [arXiv: 1905.07411] [INSPIRE]. 